\title{
STABLY TRIVIAL EQUIVARIANT ALGEBRAIC VECTOR BUNDLES
}

\author{
MIKIYA MASUDA AND TED PETRIE
}

\section{INTRODUCTION}

Let $G$ be a reductive algebraic group over $\mathbb{C}$, let $F$ be a $G$-module, and let $B$ be an affine $G$-variety, i.e., an affine variety with an algebraic action of $G$. Then $B \times F$ is in a natural way a $G$-vector bundle over $B$, which we denote by $\mathbf{F}$. (All vector bundles here are algebraic.) A $G$-vector bundle over $B$ is called trivial if it is isomorphic to $\mathbf{F}$ for some $G$-module $F$. From the endomorphism ring $R$ of the $G$-vector bundle $\mathbf{S}$, we construct $G$-vector bundles over $B$. The bundles constructed this way have the property that when added to $\mathbf{S}$ they are isomorphic to $\mathbf{F} \oplus \mathbf{S}$ for a fixed $G$-module $F$. They are called stably trivial. The set of isomorphism classes of $G$-vector bundles over $B$ which satisfy this condition is denoted by $\operatorname{VEC}(B, F ; S)$. For such a bundle $E$ we define an invariant $\rho(E)$ which lies in a quotient of $R$. This invariant allows us to distinguish non-isomorphic $G$-vector bundles. When $B$ is a $G$ module, a $G$-vector bundle over $B$ defines an action of $G$ on affine space. We give criteria which in certain cases allow us to distinguish the underlying actions. The construction and invariants are applied to the following two problems:

Equivariant Serre Problem. Is an algebraic G-vector bundle over a G-module B trivial, i.e., isomorphic to $\mathbf{F}$ for some G-module F?

Linearity Problem. Is an algebraic action of a reductive group $G$ on affine space $\mathbb{C}^{n}$ conjugate to a linear action?

Quillen and Suslin (see [Q]) showed that the Equivariant Serre Problem has an affirmative solution when $G$ is the trivial group. In particular this means that the underlying variety of a vector bundle over an affine space is itself an affine space, and hence a $G$-vector bundle over a $G$-module defines an action of $G$ on affine space-the total space of the vector bundle. This important observation is due to Bass and Haboush. See [BH1, BH2]. They show that the Equivariant Serre Problem has an affirmative solution stably. This means that any equivariant vector bundle over a $G$-module becomes trivial when added to a suitable trivial vector bundle. They also give a criterion that the action on the underlying affine space of an equivariant vector bundle over a representation is not conjugate to a linear action.

Schwarz [S] used this framework to produce the first negative solutions to both problems. His results apply when the base $G$-module has a one dimen-

Received by the editors May 4, 1993 and, in revised form, May 2, 1994.

1991 Mathematics Subject Classification. Primary 14E09, 22E50, 55M35. 
sional quotient and in that context the groups must be infinite. He used the Bass-Haboush criterion to show that in some cases there is at least one nonlinearizable action on the underlying affine spaces.

Here there is no restriction on the base. Our contributions to these problems result from specializing the results here for an arbitrary base $B$ to the case it is a $G$-module. For a variety of $G$ and $B$, we produce families of inequivalent $G$-vector bundles over $B$ and families of inequivalent non-linearizable actions of $G$ on the affine space of the underlying vector bundle. In particular we do this for several families of finite groups, producing to our knowledge the first and only negative solutions to both problems for, finite groups. Even so, finite groups play no special role in the theory or applications. Theorems A, B and C below are illustrations of this. They treat both finite and infinite groups alike.

For the purpose of illustration we discuss one of the main theorems-Theorem A. It is an application of the construction of stably trivial equivariant vector bundles and the invariant $\rho$. First we describe the basic construction of equivariant vector bundles and then state Theorem A which distinguishes them. Suppose that an affine $G$-variety $B$ has a distinguished fixed point which we denote by 0 even though $B$ need not be a vector space. The ring of invariant polynomials on $B$ is denoted $\mathscr{O}(B)^{G}$. Let $\Phi: B \rightarrow F^{*}=\operatorname{Hom}(F, \mathbb{C})$ be an algebraic equivariant map (i.e., a $G$-morphism) with $\Phi^{-1}(0)=0$. Let $T \in \mathscr{O}(B)^{G}$ be an invariant polynomial with $T(0) \neq 0$. Then the $G$-invariant hypersurface

$$
E_{\Phi}(T)=\{(x, y, z) \in B \times(F \oplus \mathbb{C}) \mid \Phi(x)(y)+T(x) z=0\}
$$

is a $G$-vector bundle over $B$ with the obvious projection on $B$. Here $\mathbb{C}$ is the trivial one dimensional $G$-module. Let $H$ be a reductive subgroup of $G$. Suppose that all units of the coordinate ring $\mathscr{O}\left(B^{H}\right)$ of $B^{H}$ are constants. An ideal $\mathscr{K}$ in $\mathscr{O}\left(B^{H}\right)$ is defined in I.4.0 (3) (take $S=\mathbb{C}$ there) whose utility is determined by

Theorem A. Let $T, T^{\prime} \in \mathscr{O}(B)^{G}$ be two invariant polynomials with $T(0)=$ $T^{\prime}(0)=1$. If $F^{H} \neq F$, then the $G$-vector bundles $E_{\Phi}(T)$ and $E_{\Phi}\left(T^{\prime}\right)$ over $B$ are isomorphic only if the restrictions of $T$ and $T^{\prime}$ to $B^{H}$ are equal modulo $\mathscr{K}$.

There is a convenient conceptual measure of the $G$-vector bundles produced by Theorem A. The set of $G$-vector bundles produced by Theorem A maps onto the image in $\mathscr{O}\left(B^{H}\right) / \mathscr{K}$ under restriction to $B^{H}$ of the set of invariant polynomials on $B$ which take the value one at 0 .

Theorem A (as well as its generalization I.4.4) leads directly to families of $G$-vector bundles over $B$ for suitable $G, B$ and choice of $F$. The families are parameterized by the invariant functions which take the value 1 at 0 . In particular Theorem A (as well as I.4.4) produces negative answers to the Equivariant Serre Problem for several families of groups. Here is a qualitative consequence of Theorem A (see Part II for more precise information):

Theorem B. There are families of inequivalent G-vector bundles over representations of the following groups: (i) infinitely many dihedral groups, (ii) infinitely 
many metacyclic groups, (iii) infinitely many subgroups of the semidirect product of the torus $\left(\mathbb{C}^{*}\right)^{q}$ and the symmetric group of degree $q$, (iv) $\mathrm{O}(2)$ and (v) $S L(2)$.

To produce negative solutions of the Linearity Problem, we need to add to Theorems A and I.4.4, the tools in $\S$ I.5. Here is a qualitative statement which is amplified precisely in Part II:

Theorem C. There are families of inequivalent actions of the groups in Theorem $\mathrm{B}$ on affine space.

For example, here is a specific case included in Theorem $\mathrm{C}$ involving the dihedral groups. Let $D_{n}$ be the dihedral group of order $2 n$ (which for $n$ equal to infinity means $O(2)$ ).

Theorem D (see II.1.5). Let $n$ be even. If $n \geq 10$, there is a non-linearizable action of $D_{n}$ on $\mathbb{C}^{4}$. If $n \geq 18$, then there is a family of inequivalent actions of $D_{n}$ on $\mathbb{C}^{4}$.

We now present an overview of the results which lead to Theorem A and I.4.4. First we show that

$$
\operatorname{VEC}(B, F ; S) \cong \operatorname{sur}(\mathbf{M}, \mathbf{S}) / \operatorname{aut}(\mathbf{M})
$$

Here $M=F \oplus S, \operatorname{sur}(\mathbf{M}, \mathbf{S})$ denotes the set of $G$-vector bundle surjections from $\mathbf{M}$ to $\mathbf{S}$, and aut $(\mathbf{M})$ denotes the group of $G$-vector bundle automorphisms of $\mathbf{M}$. This group acts on $\operatorname{sur}(\mathbf{M}, \mathbf{S})$ by right multiplication, and the right hand side of this isomorphism is the orbit space. This isomorphism is used in [MMP4] to settle the Equivariant Serre Problem for abelian groups. It is used here to define the invariant $\rho$ which is a function (see $\S \S I .1$ and I.2):

$$
\rho: \operatorname{VEC}(B, F ; S) \rightarrow(R / I)^{*} / \Gamma \text {. }
$$

Here $R$ is the ring of $G$-vector bundle endomorphisms of $\mathbf{S}, I \subset R$ is a two sided ideal in $R$ and $\Gamma$ is a subgroup of the units $(R / I)^{*}$ of $R / I$. Observe in particular that if $S$ is the trivial $G$-module $\mathbb{C}$, then $R=\mathscr{O}(B)^{G}$.

For the construction of $G$-vector bundles we take a $G$-vector bundle morphism $\Phi: \mathbf{F} \rightarrow \mathbf{S}$ and define a subset $R(\Phi)$ of $R$ and a map

$$
E_{\Phi}: R(\Phi) \rightarrow \operatorname{VEC}(B, F ; S) .
$$

In fact every stably trivial $G$-vector bundle over $B$ is $E_{\Phi}(T)$ for some $\Phi$ and $T \in R(\Phi)$. The fundamental relation between construction and invariant is

$$
\rho\left(E_{\Phi}(T)\right)=[T] \in(R / I)^{*} / \Gamma
$$

where $T \in R(\Phi)$ and $[T]$ denotes its class in the quotient.

Because of this fundamental relation, a lower bound for $\operatorname{VEC}(B, F ; S)$ is produced by solving these two problems: (1) describe $R(\Phi)$ and (2) describe $(R / I)^{*} / \Gamma$.

This paper consists of two parts. In the first part we define and develop the properties of $\rho$ and $E_{\Phi}$. Then we deal with problem (2) by mapping the complicated set $(R / I)^{*} / \Gamma$ into something simpler using the homorphism defined by 
restriction to the fixed set $B^{H}$ of a reductive subgroup $H$. This is discussed in $\S$ I.4. The culmination of the discussion is Theorem 4.4. Theorem $\mathrm{A}$ is a special case. Note that the conclusion of Theorem A involves restricting invariant functions to the fixed set of a reductive subgroup. In the setting of Theorem A, $R$ is the ring of invariant functions and the restriction homomorphism carries $R$ to $\mathscr{O}\left(B^{H}\right)$.

The previously mentioned Bass-Haboush criterion shows that a $G$-vector bundle $E$ in certain cases defines a non-linearizable action of the affine space underlying $E$. In $\S I .5$ we present criteria for distinguishing in certain cases the actions on affine space defined by $G$-vector bundles over a $G$-module $B$. When these criteria apply, they lead immediately to families of inequivalent actions of $G$ on affine space. Theorem 5.5, which is the main result, allows us to distinguish actions on affine space defined by $G$-vector bundles.

In the second part, we begin the treatment of applications which are qualitatively summarized in Theorems B and C. In particular we answer the Equivariant Serre Problem and the Linearity Problem for the groups in Theorem B. All the applications in Part II are consequences of I.4.4 (of which Theorem A is a special case) and I.5.5. In particular, the main results of application are all in Part II. In view of these theorems, i.e., I.4.4 and I.5.5, distinguishing the $G$-vector bundles constructed from $E_{\Phi}$ and the actions of $G$ on the underlying affine space they define depends on describing the ideal $\mathscr{K}$. We do this for the groups in the families mentioned above, and this yields the applications mentioned.

Historical Remarks. The results of this paper were announced in [MP1]. Because of the long delay in publishing its details here, we note some of the history and salient features of related papers treating the Equivariant Serre Problem and the Linearity Problem. The first paper producing negative responses to these two problems was [S] by Schwarz. For a certain list of infinite groups including the classical groups, he produced families of equivariant vector bundles over representations with one dimensional algebraic quotient and showed there was at least one non-linearizable action on affine space. Shortly after that, three other manuscripts appeared: our announcement [MP1], [KS] by Kraft and Schwarz, and [Kn] by Knop.

In [MP1] we assert for the groups in Theorem B here the existence of families of equivariant vector bundles over representations and families of inequivalent actions on affine space. There are both finite and infinite groups involved. We also produce criteria (see $\S 5$ here) which may be used to show that many of the actions on affine space defined by the equivariant vector bundles are distinct. This allowed us to show that for the groups in Theorem B, there are families of inequivalent actions on affine space-not just one non-linearizable action.

The details of $[\mathrm{S}]$ appeared in the manuscript [KS]. That manuscript also contained elaborations of what was in [S]. Among those were applications of the criteria (in $\S 5$ ) mentioned above. In particular, following the same line as in [MP1] and [MMP1], [KS] showed that the actions on affine space defined by some of the vector bundles in [S] and [Kn] in fact produced families of inequivalent actions. Some of these applications overlap what was previously done in [MP1] and [MMP1]. 
There is a certain overlap between [MP1] and [S], [KS], involving applications to the groups $O(2)$ and $S L(2)$. This is discussed in detail in $\S \S I I .2$ and II.5. The results in those sections dealing with equivariant vector bundles were first discussed in [S], while the results dealing with families of actions on affine space were first discussed in [MP1] and later reproduced in [KS].

The manuscript $[\mathrm{Kn}]$ uses results from [S] to show the existence of families of equivariant vector bundles over the adjoint representation of any non-abelian connected reductive group, and shows also that every group in this family has a non-linearizable action on affine space.

Finally we mention a complement to Knop's result above from our joint work with Moser-Jauslin [MMP4]. If $G$ is a reductive abelian group, any $G$-vector bundle over a representation is trivial. These to our knowledge are the only groups for which the Equivariant Serre Problem has an affirmative answer.

We are pleased to acknowledge many useful discussions with Lucy MoserJauslin.

\section{Part I. General SETting}

\section{Construction of $G$-Vector bundLes}

Let $B$ be an arbitrary affine $G$-variety. Let $F$ and $S$ be $G$-modules and $M=F \oplus S$. Denote by $\mathbf{M}, \mathbf{S}$ the trivial $G$-vector bundles over $B$ whose total spaces are $B \times M, B \times S$ respectively, by $\operatorname{mor}(\mathbf{M}, \mathbf{S})$ the set of $G$ vector bundle morphisms from $\mathbf{M}$ to $\mathbf{S}$, by $\operatorname{sur}(\mathbf{M}, \mathbf{S})$ the subset of those which are surjective on fibers, and by aut $(\mathbf{M})$ the group of $G$-vector bundle automorphisms of $\mathbf{M}$. We note that if $L \in \operatorname{sur}(\mathbf{M}, \mathbf{S})$, then its kernel $E(L)$ is a $G$-vector bundle over $B$.

Theorem 1.1. (1) If $L \in \operatorname{sur}(\mathbf{M}, \mathbf{S})$, then $E(L) \oplus \mathbf{S}$ is isomorphic to $\mathbf{M}=\mathbf{F} \oplus \mathbf{S}$ as $G$-vector bundles.

(2) If $L, L^{\prime} \in \operatorname{sur}(\mathbf{M}, \mathbf{S})$, then $E(L)$ and $E\left(L^{\prime}\right)$ are isomorphic as $G$-vector bundles if and only if there is an $A \in$ aut(M) such that $L^{\prime}=L A$.

Proof. (1) The short exact sequence of $G$-vector bundles

$$
0 \rightarrow E(L) \rightarrow \mathbf{M} \stackrel{L}{\longrightarrow} \mathbf{S} \rightarrow 0
$$

splits. This exact sequence splits non-equivariantly by a well-known result of Serre because the base is affine. (This follows from [H, III,3.7].) A result of Bass and Haboush [BH1, 4.1] asserts then that it splits equivariantly. This means that there is a $G$-vector bundle isomorphism between $\mathbf{M}$ and $E(L) \oplus \mathbf{S}$ which converts $L$ to the obvious projection of $E(L) \oplus \mathbf{S}$ to $\mathbf{S}$.

(2) Any $G$-vector bundle isomorphism from $E(L)$ to $E\left(L^{\prime}\right)$ induces a $G$ vector bundle isomorphism from $E(L) \oplus \mathbf{S}$ to $E\left(L^{\prime}\right) \oplus \mathbf{S}$ which commutes with standard projection. Using the fact that both are isomorphic to $\mathbf{M}$ then produces a $G$-vector bundle automorphism of $\mathbf{M}$ which converts $L$ to $L^{\prime}$.

The set of $G$-vector bundles over $B$ whose Whitney sum with $\mathbf{S}$ is isomorphic to $\mathbf{M}$ is denoted by $\operatorname{Vec}(B, F ; S)$, and the set of isomorphism classes of these $G$-vector bundles is denoted by $\operatorname{VEC}(B, F ; S)$. 
Corollary 1.2. Sending $L \in \operatorname{sur}(\mathbf{M}, \mathbf{S})$ to $E(L)$ defines a bijection

$$
\operatorname{sur}(\mathbf{M}, \mathbf{S}) / \operatorname{aut}(\mathbf{M}) \cong \operatorname{VEC}(B, F ; S) \text {. }
$$

This isomorphism is central to our construction of $G$-vector bundles and their invariants.

Set $R=\operatorname{end}(\mathbf{S})=\operatorname{mor}(\mathbf{S}, \mathbf{S})$. Let $\Phi \in \operatorname{mor}(\mathbf{F}, \mathbf{S})$ be fixed. Define

$$
R(\Phi)=\{T \in R \mid(\Phi, T) \in \operatorname{sur}(\mathbf{M}, \mathbf{S})\}
$$

and define

$$
E_{\Phi}: R(\Phi) \rightarrow \operatorname{VEC}(B, F ; S)
$$

by $E_{\Phi}(T)=E((\Phi, T))$ for $T \in R(\Phi)$. The map $E_{\Phi}$ produces $G$-vector bundles from the ring $R$.

Convention. In order not to proliferate notation, we often do not distinguish between a $G$-vector bundle and its isomorphism class, and use $E(L)$ either for the bundle or its isomorphism class. Similiarly $E_{\Phi}(T)$ denotes either a bundle or the isomorphism class it defines.

\section{EQUIVARIANT VECTOR BUNDLE INVARIANTS}

Let $U$ and $V$ be $G$-modules. When we wish to emphasize the source and target of a $G$-vector bundle morphism $D$ from $\mathbf{U}$ to $\mathbf{V}$, we write such a morphism as $D(U, V)$. For example, any $A \in \operatorname{aut}(\mathbf{M})$ has components $A(U, V)$ for $U=F, S$ and $V=F, S$, and similarly any $L \in \operatorname{sur}(\mathbf{M}, \mathbf{S})$ has components $L(F, S)$ and $L(S, S)$.

Let $R=\operatorname{end}(\mathbf{S})$ as before. We denote by $I$ the two sided ideal in $R$ generated by $\operatorname{mor}(\mathbf{F}, \mathbf{S}) \operatorname{mor}(\mathbf{S}, \mathbf{F})$, and the units of a ring $\Lambda$ by $\Lambda^{*}$.

Lemma 2.1. Let $A \in \operatorname{aut}(\mathbf{M})$ and $L \in$ sur. Then:

(1) $L(S, S) \in(R / I)^{*}$.

(2) $A(S, S) \in(R / I)^{*}$.

(3) $(L A)(S, S)=L(S, S) A(S, S)$ in $(R / I)^{*}$.

Proof. (1) Since $L: \mathbf{M} \rightarrow \mathbf{S}$ is surjective, there is a $G$-vector bundle morphism $P: \mathbf{S} \rightarrow \mathbf{M}$ such that $L P=1$, where 1 denotes the identity map. It follows that

$$
L(F, S) P(S, F)+L(S, S) P(S, S)=1 .
$$

Since $L(F, S) P(S, F) \in I$, this means that $P(S, S)$ is the right inverse of $L(S, S)$ in $R / I$. Note that $R$ is a finitely generated module over the commutative ring $\mathscr{O}(B)^{G}$. To see this note that the set of vector bundle endomorphisms of $\mathbf{S}$ is a finitely generated module over $\mathscr{O}(B)$. It follows from [BH1, 3.1] that $R$, the set of $G$-vector bundle endomorphisms of $\mathbf{S}$, is finitely generated over $\mathscr{O}(B)^{G}$. Since $R$ is a finitely generated module over the commutative ring $\mathscr{O}(B)^{G}$, the right inverse is also the left inverse by [M, p. 216].

(2) This follows from $A A^{-1}=A^{-1} A=1$.

(3) This follows from the identity

$$
(L A)(S, S)=L(F, S) A(S, F)+L(S, S) A(S, S) .
$$


Let

$$
\Gamma=\left\{A(S, S) \in(R / I)^{*} \mid A \in \operatorname{aut}(\mathbf{M})\right\} .
$$

One easily checks that $\Gamma$ is a subgroup of $(R / I)^{*}$.

Corollary 2.2. The map

$$
\rho: \operatorname{VEC}(B, F ; S) \rightarrow(R / I)^{*} / \Gamma,
$$

which sends $E(L) \quad(L \in \operatorname{sur}(\mathbf{M}, \mathbf{S}))$ to the class of $L(S, S)$ in $(R / I)^{*} / \Gamma$, is well defined.

Proof. This follows from 1.2 and 2.1. Then

Let $\Phi \in \operatorname{mor}(\mathbf{F}, \mathbf{S})$ and let $[T]$ denote the class of $T \in R(\Phi)$ in $(R / I)^{*} / \Gamma$.

$$
\rho\left(E_{\Phi}(T)\right)=[T] .
$$

This fundamental relation between the construction of equivariant vector bundles and the invariant distinguishing them is responsible for our results producing families of inequivalent $G$-vector bundles.

\section{NORMALIZATION}

The relation 2.3 gives a theoretical calculation of $\rho$. In general the target of $\rho$, i.e. the quotient of the group $(R / I)^{*}$ by the subgroup $\Gamma$, is complicated. In this section we begin a simplification of this target (completed in $\S 4$ ) by showing 3.4. It is possible to deal with a subgroup $\Gamma_{*}$ of $\Gamma$. This is a step in the process of removing $\Gamma$ altogether in $\S 4$. See 4.4 .

We begin with a notational remark. There is a fundamental isomorphism

$$
\operatorname{mor}(\mathbf{U}, \mathbf{V}) \cong \operatorname{Mor}(B, \operatorname{Hom}(U, V))^{G}
$$

for any two $G$-modules $U$ and $V$, where the right term in this isomorphism is the set of $G$-morphisms from $B$ to $\operatorname{Hom}(U, V)$ with its conjugation action of $G$. By definition an element $\varphi \in \operatorname{mor}(\mathbf{U}, \mathbf{V})$ is a $G$-vector bundle morphism $\varphi: \mathbf{U} \rightarrow \mathbf{V}$. As such, $\varphi(x, u)=(x, \varphi(x)(u))$ for $x \in B$ and $u \in U$. Here $\varphi(\cdot)$ is a $G$-morphism from $B$ to $\operatorname{Hom}(U, V)$. We shall freely use this correspondence between $\varphi$ and $\varphi(\cdot)$. As an important example of this identification note that

$$
\operatorname{end}(\mathbf{C})=\mathscr{O}(B)^{G} \text {. }
$$

In this section we assume that an affine $G$-variety $B$ has a distinguished fixed point, denoted 0 .

Lemma 3.1. Let $L \in \operatorname{sur}(\mathbf{M}, \mathbf{S})$. Then there exists an $L^{\prime} \in \operatorname{sur}(\mathbf{M}, \mathbf{S})$ such that $E(L)=E\left(L^{\prime}\right)$ in $\operatorname{VEC}(B, F ; S), L^{\prime}(F, S)(0)=0$ and $L^{\prime}(S, S)(0)=1$. Proof. Since the restriction of $L$ to the distinguished point 0 defines a trivial $G$-vector bundle over the point 0 , there is an automorphism $A$ of $M=F \oplus S$ which sends $L \mid 0$ to the standard projection $(0,1): F \oplus S \rightarrow S$ by 1.1 . We extend $A$ to an automorphism $A^{\prime}$ in aut(M) by setting $A^{\prime}=\pi^{*} A$, where $\pi: B \rightarrow 0$. Then $L A^{\prime}$ is the desired element $L^{\prime}$ in the lemma. 
We deduce a few corollaries. Let

$$
R(\Phi)_{*}=\{T \in R(\Phi) \mid T(0)=1\} .
$$

The following corollary follows from 1.2 and 3.1.

Corollary 3.2. Any element in $\operatorname{VEC}(B, F ; S)$ is of the form $E_{\Phi}(T)$ for some $\Phi \in \operatorname{mor}(\mathbf{F}, \mathbf{S})$ with $\Phi(0)=0$ and $T \in R(\Phi)_{*}$.

We define

$$
\begin{aligned}
\operatorname{sur}(\mathbf{M}, \mathbf{S})_{*} & =\{L \in \operatorname{sur}(\mathbf{M}, \mathbf{S}) \mid L(F, S)(0)=0, L(S, S)(0)=1\}, \\
\operatorname{aut}(\mathbf{M})_{*} & =\{A \in \operatorname{aut}(\mathbf{M}) \mid A(F, S)(0)=0, A(S, S)(0)=1\} .
\end{aligned}
$$

Note that $\operatorname{aut}(\mathbf{M})_{*}$ is a subgroup of aut $(\mathbf{M})$ acting on $\operatorname{sur}(\mathbf{M}, \mathbf{S})_{*}$ by right multiplication.

Corollary 3.3. The inclusion $\operatorname{sur}(\mathbf{M}, \mathbf{S})_{*} \rightarrow \operatorname{sur}(\mathbf{M}, \mathbf{S})$ induces a bijection

$$
\operatorname{sur}(\mathbf{M}, \mathbf{S})_{*} / \operatorname{aut}(\mathbf{M})_{*} \cong \operatorname{sur}(\mathbf{M}, \mathbf{S}) / \operatorname{aut}(\mathbf{M})(\cong \operatorname{VEC}(B, F ; S)) \text {. }
$$

Proof. The surjectivity follows from 3.1 , and the injectivity is easy.

Let

$$
\Gamma_{*}=\left\{A(S, S) \in(R / I)^{*} \mid A \in \operatorname{aut}(\mathbf{M})_{*}\right\} .
$$

Note that $\Gamma_{*}$ is also a subgroup of $(R / I)^{*}$. By 3.3 we have a version of 2.2 in this setting.

Corollary 3.4. The map

$$
\rho: \operatorname{VEC}(B, F ; S) \rightarrow(R / I)^{*} / \Gamma_{*},
$$

which sends $E(L) \quad\left(L \in \operatorname{sur}(\mathbf{M}, \mathbf{S})_{*}\right)$ to the class of $L(S, S)$ in $(R / I)^{*} / \Gamma_{*}$, is well defined.

\section{RESTRICTION}

In this section we use the composition of a restriction homomorphism and a determinant function to map the group $(R / I)^{*}$ to an abelian group $\Pi\left(\mathscr{O}\left(B^{H}\right) / \mathscr{K}\right)^{*}$. This carries the subgroup $\Gamma_{*}$ to an identifiable subgroup. Combining this with 3.4 gives a simple explicit condition (4.4) for distinguishing elements in $\operatorname{VEC}(B, F ; S)$. This condition is responsible for all our applications here in this paper.

For the remainder of this paper we make these assumptions:

Assumptions. (1) The base $B$ is a $G$-variety with distinguished fixed point denoted by 0 .

(2) We are given a reductive subgroup $H$ of $G$ such that $\mathscr{O}\left(B^{H}\right)^{*}=\mathbb{C}^{*}$. (Here $\mathbb{C}^{*}$ denotes the non-zero constant functions on $B^{H}$.)

Let $U$ and $V$ be $G$-modules. We denote by $\operatorname{Irr}(H ; U)$ the set of all (isomorphism classes of) irreducible $H$-modules which occur in $U$ viewed as an $H$-module. Let $i: B^{H} \rightarrow B$ be the inclusion. Then $i^{*} \mathbf{U}$ and $i^{*} \mathbf{V}$ are $H$-vector bundles over $B^{H}$. Since $H$ acts trivially on $B^{H}$, these $H$-vector bundles split 
into the sum of their eigensubbundles, and any $H$-vector bundle morphism between them preserves these eigensubbundles. This means

$$
\operatorname{mor}\left(i^{*} \mathbf{U}, i^{*} \mathbf{V}\right)=\prod_{\chi \in \operatorname{Irr}(H, U \oplus V)} \operatorname{mor}\left(\mathbf{U}_{\chi}, \mathbf{v}_{\chi}\right),
$$

where $U_{\chi}$ is the $\chi$ isotypical component of $U$ viewed as an $H$-module and $\mathbf{U}_{\chi}$ is the $\chi$ eigensubbundle of $i^{*} \mathbf{U}$. If $D \in \operatorname{mor}(\mathbf{U}, \mathbf{V})$, then its restriction $i^{*} D$ over $B^{H}$ is an $H$-vector bundle morphism from $i^{*} \mathbf{U}$ to $i^{*} \mathbf{V}$. Let $D_{\chi}$ denote its restriction to the $\chi$ eigensubbundle. Thus we have a map

$$
i^{*}: \operatorname{mor}(\mathbf{U}, \mathbf{V}) \rightarrow \operatorname{mor}\left(i^{*} \mathbf{U}, i^{*} \mathbf{V}\right),
$$

and in particular

$$
i^{*}: R=\operatorname{end}(\mathbf{S}) \rightarrow \operatorname{end}\left(i^{*} \mathbf{S}\right)=\prod_{\chi \in \operatorname{Irr}(H ; S)} \operatorname{end}\left(\mathbf{S}_{\chi}\right) .
$$

The determinant function det $: \operatorname{end}\left(\mathbf{S}_{\chi}\right) \rightarrow \mathscr{O}\left(B^{H}\right)$ extends componentwise to give

$$
\text { Det }: \operatorname{end}\left(i^{*} \mathbf{S}\right) \rightarrow \prod \mathscr{O}\left(B^{H}\right),
$$

where the product is over all $\chi \in \operatorname{Irr}(H ; S)$.

As noted before,

$$
\operatorname{mor}\left(i^{*} \mathbf{U}, i^{*} \mathbf{V}\right)=\operatorname{Mor}\left(B^{H}, \operatorname{Hom}(U, V)\right)^{H},
$$

so each $P \in \operatorname{mor}\left(i^{*} \mathbf{U}, i^{*} \mathbf{V}\right)$ is represented by a matrix with entries in $\mathscr{O}\left(B^{H}\right)$. Let $(P)$ denote the ideal in $\mathscr{O}\left(B^{H}\right)$ generated by the coefficients of the matrix representation of $P$. If $Y \subset \operatorname{mor}\left(i^{*} \mathbf{U}, i^{*} \mathbf{V}\right)$, let $(Y)$ be the ideal $\sum_{P \in Y}(P)$. In particular, we set

$$
\left(i^{*} \operatorname{mor}(\mathbf{U}, \mathbf{V})\right)=J(U, V) .
$$

We now define the ideal $\mathscr{K} \subset \mathscr{O}\left(B^{H}\right)$. The following convention is adopted: If $D \in \operatorname{end}(\mathbf{U})$, then $\operatorname{det} D_{\chi}=1$ whenever $\chi$ is an irreducible $\dot{H}$-module not in $\operatorname{Irr}(H ; U)$. As is the previous sections, $M=F \oplus S$. The ideals defined below depend on $B$ and $H$, but in order to avoid unnessecary notation, $B$ and $H$ are omitted in the notation.

Definition 4.0. (1) end $(\mathbf{F} ; \mathbf{S}) \subset \operatorname{end}(\mathbf{F})$ consists of those $D \in \operatorname{end}(\mathbf{F})$ such that $\operatorname{det} D_{\chi} \in \mathbb{C}^{*}$ whenever $\chi \in \operatorname{Irr}(H ; F)-\operatorname{Irr}(H ; S)$.

(2) $D(F ; S)$ is the ideal in $\mathscr{O}\left(B^{H}\right)$ generated by

$$
\left\{D_{\chi, \chi^{\prime}} \mid D \in \operatorname{end}(\mathbf{F} ; \mathbf{S}), \quad \chi, \chi^{\prime} \in \operatorname{Irr}(H ; M)\right\}
$$

where $D_{\chi, \chi^{\prime}}=\left(\operatorname{det} D_{\chi^{\prime}}\right)(0) \operatorname{det} D_{\chi}-\left(\operatorname{det} D_{\chi}\right)(0) \operatorname{det} D_{\chi^{\prime}}$ and $\operatorname{det} D_{\chi}$ is understood to be 1 unless $\chi \in \operatorname{Irr}(H ; F)$.

(3) $\mathscr{K}$ is the ideal $J(F, S) J(S, F)+D(F ; S)$ in $\mathscr{O}\left(B^{H}\right)$.

Since $\left(i^{*} I\right) \subset J(F, S) J(S, F) \subset \mathscr{K}$, Det maps $i^{*} I$ into $\prod \mathscr{K} \subset \prod \mathscr{O}\left(B^{H}\right)$, where the product is over all $\chi \in \operatorname{Irr}(H ; S)$. This means that the restriction 
map $i^{*}$ composed with Det induces a map

$$
\operatorname{Det} i^{*}:(R / I)^{*} \rightarrow \prod_{\chi \in \operatorname{Irr}(H ; S)}\left(\mathscr{O}\left(B^{H}\right) / \mathscr{K}\right)^{*}
$$

In order to use 3.4, we must describe the image of $\Gamma_{*} \subset(R / I)^{*}$ under this map. We begin this process.

Lemma 4.1. If $A \in \operatorname{aut}(\mathbf{M})$, then $A(F, F) \in \operatorname{end}(\mathbf{F} ; \mathbf{S})$.

Proof. Since $A$ is an automorphism, $\operatorname{det} A_{\chi} \in \mathscr{O}\left(B^{H}\right)^{*}=\mathbb{C}^{*}$ for each $\chi \in$ $\operatorname{Irr}(H ; M)$. If $\chi \in \operatorname{Irr}(H ; F)-\operatorname{Irr}(H ; S)$, then $A_{\chi}=A(F, F)_{\chi}$. Hence $\operatorname{det} A(F, F)_{\chi} \in \mathbb{C}^{*}$ for such $\chi$, as required.

Lemma 4.2. If $A \in \operatorname{aut}(\mathbf{M})$, then

$$
\operatorname{det} A(S, S)_{\chi} \equiv \operatorname{det} A_{\chi} \operatorname{det} A^{-1}(F, F)_{\chi} \bmod J(F, S) J(S, F)
$$

for each $\chi \in \operatorname{Irr}(H ; M)$.

Proof. If $\chi \in \operatorname{Irr}(H ; F)-\operatorname{Irr}(H ; S)$, then $\operatorname{det} A(S, S)_{\chi}=1$ by the convention introduced just before 4.0, and $A_{\chi}^{-1}=A^{-1}(F, F)_{\chi}$. Therefore the congruence (in fact, the identity) holds in this case. If $\chi \in \operatorname{Irr}(H ; S)-\operatorname{Irr}(H ; F)$, then $\operatorname{det} A^{-1}(F, F)_{\chi}=1$ by the convention, and $A(S, S)_{\chi}=A_{\chi}$. Therefore the congruence holds in this case, too.

In the following we assume $\chi \in \operatorname{Irr}(H ; F) \cap \operatorname{Irr}(H ; S)$. By definition, $A(F, S)_{\chi}$ is the $\chi$ component of $i^{*} A(F, S)$; so $\left(A(F, S)_{\chi}\right) \subset\left(i^{*} A(F, S)\right) \subset$ $J(F, S)$. Likewise, $\left(A(S, F)_{\chi}\right) \subset J(S, F)$. Hence we have

$$
\operatorname{det} A(F, F)_{\chi} \operatorname{det} A(S, S)_{\chi} \equiv \operatorname{det} A_{\chi} \bmod J(F, S) J(S, F) .
$$

On the other hand, it follows from $A_{\chi}\left(A^{-1}\right)_{\chi}=1$ that

$$
A(F, F)_{\chi} A^{-1}(F, F)_{\chi}+A(S, F)_{\chi} A^{-1}(F, S)_{\chi}=1 .
$$

Since $\left(A(S, F)_{\chi} A^{-1}(F, S)_{\chi}\right) \subset J(F, S) J(S, F)$, we have

$$
\operatorname{det} A(F, F)_{\chi} \operatorname{det} A^{-1}(F, F)_{\chi} \equiv 1 \bmod J(F, S) J(S, F) .
$$

The lemma follows from the two congruences above.

Lemma 4.3. Let $A \in \operatorname{aut}(\mathbf{M})_{*}$. Then:

(1) $\operatorname{det} A(S, S)_{\chi}$ modulo $\mathscr{K}$ is independent of $\chi \in \operatorname{Irr}(H ; M)$.

(2) If $\operatorname{Irr}(H ; F) \neq \operatorname{Irr}(H ; S)$, then $\operatorname{det} A(S, S)_{\chi} \equiv 1 \bmod \mathscr{K}$ for all $\chi \in$ $\operatorname{Irr}(H ; M)$.

Proof. (1) Let $d=\operatorname{det} A^{-1}(F, F), d_{\chi}=\operatorname{det} A^{-1}(F, F)_{\chi}$ for $\chi \in \operatorname{Irr}(H ; M)$. Since $A^{-1} \in \operatorname{aut}(\mathbf{M})_{*}, \operatorname{det} A_{\chi}^{-1}$ is a non-zero constant and equal to $d_{\chi}(0)$ (remember that $\left.A^{-1}(S, S)(0)=1\right)$. Hence $\operatorname{det} A_{\chi}=d_{\chi}(0)^{-1}$. On the other 
hand $A^{-1}(F, F) \in \operatorname{end}(\mathbf{F} ; \mathbf{S})$ by 4.1 ; so $d_{\chi^{\prime}}(0) d_{\chi}-d_{\chi}(0) d_{\chi^{\prime}} \in D(F ; S)$ for each $\chi, \chi^{\prime} \in \operatorname{Irr}(H ; M)$, which implies

$$
d_{\chi} / d_{\chi}(0) \equiv d_{\chi^{\prime}} / d_{\chi^{\prime}}(0) \bmod \mathscr{K}
$$

This together with 4.2 proves the statement (1), since $d_{\chi}(0)^{-1}=\operatorname{det} A_{\chi}$ and $d_{\chi}=\operatorname{det} A^{-1}(F, F)_{\chi}$ for any $\chi \in \operatorname{Irr}(H ; M)$.

(2) If $\operatorname{Irr}(H ; F) \neq \operatorname{Irr}(H ; S)$, then there are two cases:

(i) There is a $\mu$ in $\operatorname{Irr}(H ; F)$ but not in $\operatorname{Irr}(H ; S)$.

(ii) There is a $\mu$ in $\operatorname{Irr}(H ; S)$ but not in $\operatorname{Irr}(H ; F)$.

In case (i), $\operatorname{det} A(S, S)_{\mu}=1$ by convention. In case (ii), $A(S, S)_{\mu}=A_{\mu}$, so $\operatorname{det} A(S, S)_{\mu}=\operatorname{det} A_{\mu}$ is a non-zero constant because $A$ is an automorphism. On the other hand, $A(S, S)(0)=1$ since $A \in \operatorname{aut}(\mathbf{M})_{*}$; hence $\operatorname{det} A(S, S)_{\mu}=1$ in this case, too. This together with (1) proves (2).

Lemma 4.3 says that the image of $\Gamma_{*} \subset(R / I)^{*}$ by the map

$$
\operatorname{Det} i^{*}:(R / I)^{*} \rightarrow \prod_{\chi \in \operatorname{Irr}(H ; S)}\left(\mathscr{O}\left(B^{H}\right) / \mathscr{K}\right)^{*}
$$

is contained in the diagonal subgroup and trivial if $\operatorname{Irr}(H ; F) \neq \operatorname{Irr}(H ; S)$. By 3.2 any element in $\operatorname{VEC}(B, F ; S)$ is of the form $E_{\Phi}(T)$ for some $\Phi \in$ $\operatorname{mor}(\mathbf{F}, \mathbf{S})$ with $\Phi(0)=0$ and $T \in R(\Phi)_{*}$. By definition $\rho\left(E_{\Phi}(T)\right)$ is the class of $T$ in $(R / I)^{*} / \Gamma_{*}$. Since $\operatorname{Det} i^{*} T=\prod \operatorname{det} T_{\chi}$, we obtain

Theorem 4.4. Let $\Phi, \Phi^{\prime} \in \operatorname{mor}(\mathbf{F}, \mathbf{S})$, where $\Phi(0)=0=\Phi^{\prime}(0)$ and $T \in R(\Phi)_{*}$ and $T^{\prime} \in R\left(\Phi^{\prime}\right)_{*}$. Suppose that $G$-vector bundles $E_{\Phi}(T)$ and $E_{\Phi^{\prime}}\left(T^{\prime}\right)$ are isomorphic. Then, if $\operatorname{Irr}(H ; F) \neq \operatorname{Irr}(H ; S)$,

$$
\operatorname{det} T_{\chi}^{\prime} \equiv \operatorname{det} T_{\chi} \bmod \mathscr{K} \quad \text { for all } \chi \in \operatorname{Irr}(H ; S) \text {, }
$$

and otherwise

$$
\operatorname{det} T_{\chi}^{\prime} \operatorname{det} T_{\chi^{\prime}} \equiv \operatorname{det} T_{\chi^{\prime}}^{\prime} \operatorname{det} T_{\chi} \quad \bmod \mathscr{K} \quad \text { for all } \chi, \chi^{\prime} \in \operatorname{Irr}(H ; S) \text {. }
$$

Proof of Theorem $A$. To convert a special case of 4.4 to Theorem A, take $S=\mathbb{C}$ and $\Phi=\Phi^{\prime}$. Since $\mathbb{C}$ is one dimensional, there is only one $\chi$ in $\operatorname{Irr}(H ; S)$ and $i^{*} T=T_{\chi}=\operatorname{det} T_{\chi}$. Hence the conclusion of 4.4 becomes $i^{*} T \equiv i^{*} T^{\prime}$ $\bmod \mathscr{K}$, which is the conclusion of Theorem $\mathrm{A}$.

\section{Distinguishing $G$-varieties}

In this section $B$ is a $G$-module. We denote by $\operatorname{Aut}(B)^{G}$ the group of $G$ variety automorphisms of $B$. From the solution of the Serre conjecture by Quillen and Suslin, every $G$-vector bundle $E$ over $B$ is trivial when we forget the action, so $E$ defines an action of $G$ on affine space. In this section we discuss how to distinguish actions of $G$ defined by $G$-vector bundles over $B$. There are already two related results in this direction ([BH2], [Kr]).

Note that for any $G$-vector bundle over $B$ the fiber over a fixed point in $B$ is a $G$-module, and the isomorphism class of this $G$-module is independent 
of the fixed point because the fixed point set is connected. From this one sees easily that two $G$-vector bundles over $B$ which are isomorphic as $G$-varieties have isomorphic $G$-modules on the fibers over 0 . Since $G$-vector bundles over $B$ are stably trivial, any two $G$-vector bundles over $B$ which are isomorphic as $G$-varieties lie in $\operatorname{Vec}(B, F ; S)$ for some $G$-module $S$, where $F$ is the common $G$-module on the zero fiber.

The following results are applied in later sections to exhibit families of actions of $G$ contructed from $G$-vector bundles.

Lemma 5.1. Let $E, E^{\prime}$ be G-vector bundles over $B$. Let $l: B \rightarrow E$ be the zero section and $p: E^{\prime} \rightarrow B$ be the projection. Then there is a G-variety isomorphism $\psi: E \rightarrow E^{\prime}$ such that

$$
p \psi l \in \operatorname{Aut}(B)^{G}
$$

if and only if there is an $f \in \operatorname{Aut}(B)^{G}$ such that $E$ and $f^{*} E^{\prime}$ are isomorphic as $G$-vector bundles.

Proof. The "if" part is trivial, so we prove the "only if" part.

Step 1 . Suppose $\psi$ preserves the zero section $B$. Then the normal derivative of $\psi$ at $B$ gives a $G$-vector bundle isomorphism of $E$ and $(p \psi l)^{*} E^{\prime}$.

Step 2. We use the fact that $E, E^{\prime} \in \operatorname{Vec}(B, F ; S)$ for some $S$ as noted above. Let $x \in B$ and $w \in M=F \oplus S$. We write

$$
\psi(x, w)=\left(\psi_{1}(x, w), \psi_{2}(x, w)\right) \text { for }(x, w) \in E \subset B \times M
$$

where $\psi_{1}(x, w) \in B$ and $\psi_{2}(x, w) \in M$. By the assumption, $\psi_{1}(, 0)=$ $p \psi \imath \in \operatorname{Aut}(B)^{G}$. We define a $G$-variety automorphism $\varphi$ of $E^{\prime}$ by

$$
\varphi\left(\psi_{1}(x, 0), w\right)=\left(\psi_{1}(x, 0), w+\psi_{2}(x, 0)\right) .
$$

Since $\psi(B)=\varphi(B)$, the composition $\varphi^{-1} \psi: E \rightarrow E^{\prime}$ is a $G$-variety isomorphism which preserves the zero section $B$. Thus Step 1 can be applied, and the lemma follows.

Corollary 5.2. Let $E, E^{\prime}$ be $G$-vector bundles over $B$ with the same $G$-module $F$ on the zero fiber. Suppose $G$ contains a subgroup $C$ such that $(B \oplus F)^{C}=B$. Then $E$ and $E^{\prime}$ are isomorphic as $G$-varieties if and only if $E$ and $f^{*} E^{\prime}$ are isomorphic as $G$-vector bundles for some $f \in \operatorname{Aut}(B)^{G}$.

Proof. By previous remarks we may suppose that $E, E^{\prime} \in \operatorname{Vec}(B, F ; S)$. The assumption means that $B^{C}=B$ and $F^{C}=0$. Since $E^{C}$ is a vector bundle over $B^{C}$ with fiber $F^{C}$, it follows that $E^{C}=B=\left(E^{\prime}\right)^{C}$; so any $G$-variety isomorphism $E \rightarrow E^{\prime}$ induces a $G$-variety automorphism of $B$ and $5.1(*)$ is satisfied. The "only if" part then follows from 5.1. The "if" part is trivial.

Note that $\mathbb{C}^{*}$ acts on $E$ by scalar multiplication on the fibers, so $E$ admits an action of $G \times \mathbb{C}^{*}$. For any integer $k \geq 1$ we define $G_{k}=G \times \mathbb{Z} / k \mathbb{Z}$ and regard it as a subgroup of $G \times \mathbb{C}^{*}$ in a natural way. 
Corollary 5.3. Let $E$ and $E^{\prime}$ be $G$-vector bundles over $B$. For any $k \geq 2, E$ and $E^{\prime}$ are isomorphic as $G_{k}$-varieties if and only if $E$ and $f^{*} E^{\prime}$ are isomorphic as $G$-vector bundles for some $f \in \operatorname{Aut}(B)^{G}$.

Proof. Since $k \geq 2, E^{\mathbb{Z} / k \mathbb{Z}}=\left(E^{\prime}\right)^{\mathbb{Z} / k \mathbb{Z}}=B$. Hence any $G_{k}$-variety isomorphism $E \rightarrow E^{\prime}$ induces a $G$-variety automorphism of $B$. Thus the "only if" part follows from 5.1. The "if" part is trivial.

The reader may wonder whether the corollary above holds even when $k=1$. This is often the case, as is seen in 5.2. But the following example shows that it is not always the case.

Example. Suppose $B=B^{\prime} \times S$ for some $G$-module $B^{\prime}$ and $\operatorname{VEC}\left(B^{\prime}, F ; S\right)$ is not trivial. Let $\pi: B=B^{\prime} \times S \rightarrow B^{\prime}$ be the projection. It induces an injection

$$
\pi^{*}: \operatorname{VEC}\left(B^{\prime}, F ; S\right) \rightarrow \operatorname{VEC}(B, F ; S)
$$

because there is a natural inclusion map $l: B^{\prime} \rightarrow B$ such that $l^{*} \pi^{*}$ is the identity. In particular, if $E \in \operatorname{VEC}\left(B^{\prime}, F ; S\right)$ is non-trivial, then so is $\pi^{*} E \in$ $\operatorname{VEC}(B, F ; S)$ and hence there is no element $f \in \operatorname{Aut}(B)^{G}$ such that $\pi^{*} E=$ $f^{*} \mathbf{F}$. On the other hand, $\pi^{*} E$ is isomorphic to $\mathbf{F}$ as $G$-varieties since we have $G$-variety isomorphisms

$$
\pi^{*} E \cong E \times S \cong E \oplus\left(B^{\prime} \times S\right) \cong B^{\prime} \times(F \oplus S) \cong B \times F=\mathbf{F},
$$

where the third isomorphism is because $E$ is in $\operatorname{VEC}\left(B^{\prime}, F ; S\right)$.

The example above means that $5.1\left(^{*}\right)$ is not necessarily satisfied. We shall give another sufficient condition for $5.1\left(^{*}\right)$ to hold for any $G$-variety isomorphism $\psi$.

Lemma 5.4. Suppose that $\operatorname{dim} B \geq 2$ and $\operatorname{Mor}(B, B)^{G}$ is generated by the identity map as an $\mathscr{O}(B)^{G}$-module. Suppose that there exists a map $\omega: B \rightarrow B$ such that

(1) $\varphi \omega=\varphi$ for all $\varphi \in \operatorname{Mor}(B, V)^{G}$, where $V=\mathbb{C}, F$,

(2) $\omega(x) \neq x$ for all $x \neq 0 \in B$.

Then $5.1(*)$ is satisfied for any $G$-variety isomorphism $\psi$.

Proof. Since $\operatorname{Mor}(B, B)^{G}$ is generated by the identity map as an $\mathscr{O}(B)^{G}$ module, one can write

$$
\psi(x, 0,0)=(\alpha(x) x, \beta(x), \gamma(x)) \in E^{\prime} \subset B \times(F \oplus S)
$$

with $\alpha \in \mathscr{O}(B)^{G}=\operatorname{Mor}(B, \mathbb{C})^{G}, \beta \in \operatorname{Mor}(B, F)^{G}, \gamma \in \operatorname{Mor}(B, S)^{G}$. By 3.2 we may assume $E^{\prime}=E_{\Phi}(T)$ for some $\Phi \in \operatorname{mor}(\mathbf{F}, \mathbf{S})$ with $\Phi(0)=0$ and $T \in R(\Phi)_{*}$, so

$$
\Phi(\alpha(x) x)(\beta(x))+T(\alpha(x) x)(\gamma(x))=0 .
$$

Suppose $\alpha$ is not a non-zero constant. Then there is a non-zero root $x^{\prime}$ of $\alpha=0$, as $\operatorname{dim} B \geq 2$. Since $\Phi(0)=0$ and $T(0)=1$, it follows from the identity above and (1) that $\gamma\left(x^{\prime}\right)=0=\gamma\left(\omega\left(x^{\prime}\right)\right)$. Therefore $\psi\left(x^{\prime}, 0,0\right)=$ 
$\psi\left(\omega\left(x^{\prime}\right), 0,0\right)$, again by (1). Since $\psi$ is an isomorphism and, in particular, injective, we have $x^{\prime}=\omega\left(x^{\prime}\right)$. But this contradicts (2) as $x^{\prime} \neq 0$. Therefore $\alpha$ must be a non-zero constant, and this verifies the assumption in 5.1.

Now the question becomes when we can find enough information about $\operatorname{Aut}(B)^{G}$ to use these results. Since $B$ is a $G$-module, multiplication by a non-zero complex number defines a $G$-variety automorphism of $B$; so $\mathbb{C}^{*}$ is isomorphic to a subgroup of $\operatorname{Aut}(B)^{G}$. We sometimes regard $\mathbb{C}^{*}$ as a subgroup of $\operatorname{Aut}(B)^{G}$ this way. If $\operatorname{Mor}(B, B)^{G}$ is generated by the identity map as an $\mathscr{O}(B)^{G}$-module (cf. 5.4), then $\operatorname{Aut}(B)^{G}=\mathbb{C}^{*}$, but it is difficult to compute $\operatorname{Aut}(B)^{G}$ in general. The next theorem gives a way to avoid this difficulty.

We note that $\operatorname{Aut}(B)^{G}$ acts on $\operatorname{mor}(\mathbf{F}, \mathbf{S})$, etc., through its action on $B$. The action of $f \in \operatorname{Aut}(B)^{G}$ on $\Phi \in \operatorname{mor}(\mathbf{F}, \mathbf{S})$, etc., then is denoted $f^{*} \Phi$. Similarly $\operatorname{Aut}\left(B^{H}\right)$ acts on $\mathscr{O}\left(B^{H}\right)$. The inclusion map $i: B^{H} \rightarrow B$ induces a homomorphism $i^{*}: \operatorname{Aut}(B)^{G} \rightarrow \operatorname{Aut}\left(B^{H}\right)$. We remind the reader that $G_{k}$ is the group $G \times \mathbb{Z} / k \mathbb{Z}$ and $\mathscr{K}$ is the ideal defined in 4.0.

Theorem 5.5. Let $\Phi, \Phi^{\prime} \in \operatorname{mor}(\mathbf{F}, \mathbf{S})$ satisfy $\Phi(0)=0=\Phi^{\prime}(0)$, and let $T \in$ $R(\Phi)_{*}, T^{\prime} \in R\left(\Phi^{\prime}\right)_{*}$. Let $H$ be a reductive subgroup of $G$. Suppose that there is a $G_{k^{-}}$-variety isomorphism $\psi: E_{\Phi}(T) \rightarrow E_{\Phi^{\prime}}\left(T^{\prime}\right)$ where either $k \geq 2$ or $\psi$ satisfies $5.1(*)$. Then there is a $c \in i^{*}\left(\operatorname{Aut}(B)^{G}\right) \subset \operatorname{Aut}\left(B^{H}\right)$ such that in case $\operatorname{Irr}(H ; F) \neq \operatorname{Irr}(H ; S)$

$$
c^{*} \operatorname{det} T_{\chi}^{\prime} \equiv \operatorname{det} T_{\chi} \bmod \mathscr{K}
$$

for all $\chi \in \operatorname{Irr}(H ; S)$, and otherwise

$$
\left(c^{*} \operatorname{det} T_{\chi}^{\prime}\right) \operatorname{det} T_{\chi^{\prime}} \equiv\left(c^{*} \operatorname{det} T_{\chi^{\prime}}^{\prime}\right) \operatorname{det} T_{\chi} \bmod \mathscr{K}
$$

for all $\chi, \chi^{\prime} \in \operatorname{Irr}(H ; S)$.

Proof. The assumption that $k \geq 2$ or $\psi$ satisfies $5.1\left(^{*}\right)$ means that 5.1 or 5.3 applies to give an automorphism $f \in \operatorname{Aut}(B)^{G}$ such that $E_{\Phi}(T)$ is isomorphic to $f^{*} E_{\Phi^{\prime}}\left(T^{\prime}\right)$ as $G$-vector bundles. Note $f^{*} E_{\Phi^{\prime}}\left(T^{\prime}\right)=E_{f^{*} \Phi^{\prime}}\left(f^{*} T^{\prime}\right)$. Since $f$ restricts to an automorphism $c$ of $B^{H}$, the theorem follows from 4.4.

Remark. In later applications $B^{G}=\{0\}$ and $B^{H}$ is one dimensional, so $i^{*}\left(\operatorname{Aut}(B)^{G}\right)=\mathbb{C}^{*}$.

We end this section with

Lemma 5.6. Let $T \in R(\Phi)_{*}$ and $c \in \mathbb{C}^{*} \subset \operatorname{Aut}(B)^{G}$. If $\Phi$ is homogeneous, then $c^{*} E_{\Phi}(T) \cong E_{\Phi}\left(c^{*} T\right)$ as $G$-vector bundles and $E_{\Phi}(T) \cong E_{\Phi}\left(c^{*} T\right)$ as $G_{k}$-varieties for any $k \geq 1$.

Proof. First we note that $c^{*} E_{\Phi}(T)=E_{c^{*} \Phi}\left(c^{*} T\right)$. Since $\Phi$ is homogeneous, $c^{*} \Phi=c^{\operatorname{deg} \Phi} \Phi$. Through an automorphism $(y, z) \rightarrow\left(c^{\operatorname{deg} \Phi} y, z\right)$ of $F \oplus S$, one sees that $E_{c^{*} \Phi}\left(c^{*} T\right) \cong E_{\Phi}\left(c^{*} T\right)$ as $G$-vector bundles. This proves the first statement of the lemma. 
Since $c^{*} E_{\Phi}(T) \cong E_{\Phi}\left(c^{*} T\right)$ as $G$-vector bundles, they are isomorphic as $G_{k}$ varieties. Here $c^{*} E_{\Phi}(T)$ is isomorphic to $E_{\Phi}(T)$ as $G_{k}$-varieties because it is the pullback of $E_{\Phi}(T)$ by $c \in \operatorname{Aut}(B)^{G}$. This proves the latter statement of the lemma.

\section{PART II. ApPlications}

In this part of the paper we present applications of the material in Part I. For certain groups, we explicitly construct families of $G$-vector bundles over $G$-modules and distinguish them up to isomorphism as $G$-vector bundles and as $G$-varieties. (These $G$-vector bundles define actions on affine space, and the actions defined on affine space by two $G$-vector bundles are distinct if and only if the $G$-vector bundles are distinct as $G$-varieties.) The key tools are I.4.4 and I.5.5 for $G$-vector bundles and $G$-varieties respectively. Three families of finite groups are treated. In addition, applications to $O(2)$ and $S L(2)$ are included, illustrating that the methods of Part I apply equally to finite and infinite reductive groups. The section on $S L(2)$ serves also as a preview of applications to semisimple groups in [MMP1] and [MMP2].

In the applications the base space $B$ is a $G$-module, so it has the origin as a distinguished fixed point and $\mathscr{O}\left(B^{H}\right)^{*}=\mathbb{C}^{*}$ for any reductive subgroup $H$ of $G$. Therefore the assumptions in $\S I .4$ are satisfied. Our applications treat the $G$-vector bundles $E_{\Phi}(T) \in \operatorname{VEC}(B, F ; S)$ as $T$ varies in $R(\Phi)_{*}$ for a fixed $\Phi \in \operatorname{mor}(\mathbf{F}, \mathbf{S})$. We choose $\Phi$, describe $R(\Phi)_{*}$, and distinguish $E_{\Phi}(T)$ and $E_{\Phi}\left(T^{\prime}\right)$ as $G$-vector bundles and as $G$-varieties. To use I.4.4 to distinguish $G$ vector bundles we choose $\Phi=\Phi^{\prime} \in \operatorname{mor}(\mathbf{F}, \mathbf{S})$ to be homogeneous of positive degree (explanation below) so that $\Phi(0)=0$. In each application we choose a subgroup $H$ and show that $\mathscr{K} \subset \mathscr{O}\left(B^{H}\right)_{e}$ for a certain $e$ depending on the data, where $\mathscr{O}\left(B^{H}\right)_{e}$ denotes the ideal in $\mathscr{O}\left(B^{H}\right)$ generated by homogeneous elements of degree $\geq e$.

Remember that $\varphi \in \operatorname{mor}(\mathbf{U}, \mathbf{V})$ determines and is determined by the $G$ morphism $\varphi(\cdot): B \rightarrow \operatorname{Hom}(U, V)$ defined by $\varphi(x, u)=(x, \varphi(x)(u))$ for $x \in B$ and $u \in U$. In this way $\varphi$ is viewed as a morphism between vector spaces. Its degree is the minimal degree of its coordinate functions, and it is homogeneous of degree $d$ if $\varphi(c x)=c^{d} \varphi(x)$ for $c \in \mathbb{C}$ and $x \in B$. We often use a matrix expression for $\varphi(x)$ in which elements in $U$ and $V$ are regarded as column vectors. Remember also that for a (non-zero) constant $c \in \mathbb{C}, c^{*} \varphi$ denotes the morphism in $\operatorname{mor}(\mathbf{U}, \mathbf{V})$ defined by $c^{*} \varphi(x)=\varphi(c x)$ for $x \in B$.

\section{DihedRal GROUPS}

Let $K=\mathbb{Z} / n \mathbb{Z}$. We fix a generator of $K$ and denote it by $g$. Let $U_{r}$ be the one dimensional $K$-module given by $g u=\zeta^{r} u$, where $u \in U_{r}=\mathbb{C}$ and $\zeta=\exp (2 \pi \sqrt{-1} / n)$. In this section $G$ is the dihedral group $D_{n}$ of order $2 n$, that is, a semidirect product of $K=\mathbb{Z} / n \mathbb{Z}$ and $H=\mathbb{Z} / 2 \mathbb{Z}$. We denote by $h$ the non-trivial element in $H$. Let $V_{r}$ be the two dimensional $G$-module defined by

$$
g(u, v)=\left(\zeta^{r} u, \zeta^{-r} v\right), \quad h(u, v)=(v, u),
$$


where $(u, v) \in V_{r}=\mathbb{C}^{2}$. Note that $V_{r}=U_{r} \oplus U_{-r}$ as $K$-modules.

We take $B=V_{l}$ and denote the coordinate of $B$ by $(a, b)$. Then $B^{H}=$ $\{(a, b) \in B \mid a=b\}$. We must estimate the ideal $\mathscr{K}$ in $\mathscr{O}\left(B^{H}\right)$ defined in 4.0. To do this we introduce some notation.

Definition. With integers $l, r$ we associate a non-negative integer

$$
[l, r ; G]=\min \{i+j \mid l(i-j) \equiv r \bmod n, i \geq 0, j \geq 0\} .
$$

Lemma 1.1. Any (homogeneous) element of $\operatorname{Mor}\left(U_{l} \oplus U_{-l}, U_{r}\right)^{K}$ has degree $\geq[l, r ; G]$. In particular, any (homogeneous) element of $\operatorname{Mor}\left(V_{l}, V_{r}\right)^{G}$ has degree $\geq[l, r ; G]$.

Proof. Let $(a, b) \in U_{l} \oplus U_{-l}$ and let $f \in \operatorname{Mor}\left(U_{l} \oplus U_{-l}, U_{r}\right)^{K}$. The equivariance relative to $K$ implies that $f\left(\zeta^{l} a, \zeta^{-l} b\right)=\zeta^{r} f(a, b)$. Suppose a non-zero scalar multiple of $a^{i} b^{j} \quad(i, j \geq 0)$ appears in $f(a, b)$. Then, comparing the coefficients of $a^{i} b^{j}$ in the identity above, we have $\zeta^{l(i-j)}=\zeta^{r}$, i.e., $l(i-j) \equiv r$ $\bmod n$. It follows from the definition of $[l, r ; G]$ that $i+j \geq[l, r ; G]$. This proves the lemma.

Lemma 1.2. Let $B=V_{l}, F=V_{m}, S=\mathbb{C}$ or $V_{s}$. Then

(1) $J(S, F)=J(F, S) \subset \mathscr{O}\left(B^{H}\right)_{d}$, where $d=[l, m ; G]$ when $S=\mathbb{C}$ and $d=\min \{[l, m-s ; G],[l, m+s ; G]\}$ when $S=V_{s}$.

(2) $D(F ; S) \subset \mathscr{O}\left(B^{H}\right)_{[l, 2 m ; G]}$.

Hence $\mathscr{K} \subset \mathscr{O}\left(B^{H}\right)_{e}$, where $e=\min \{2 d,[l, 2 m ; G]\}$ and $d$ is as in (1).

Proof. (1) Since $F$ and $S$ are both self-dual, $\operatorname{mor}(\mathbf{S}, \mathbf{F})$ and $\operatorname{mor}(\mathbf{F}, \mathbf{S})$ are isomorphic, which implies that $J(S, F)=J(F, S)$. If $S=\mathbb{C}, \operatorname{mor}(\mathbf{S}, \mathbf{F}) \cong$ $\operatorname{Mor}\left(V_{l}, V_{m}\right)^{G}$; so the desired estimate follows from 1.1. If $S=V_{s}$, then $\operatorname{Hom}(S, F) \cong V_{s}^{*} \otimes V_{m} \cong V_{m-s} \oplus V_{m+s}$; so the desired estimate follows again from 1.1 .

(2) Let $D \in \operatorname{end}(\mathbf{F})$. It follows from the equivariance relative to $H$ that $D$ is of the form

$$
D(a, b)=\left(\begin{array}{ll}
p_{1}(a, b) & p_{2}(a, b) \\
p_{2}(b, a) & p_{1}(b, a)
\end{array}\right)
$$

where $p_{i}(i=1,2)$ are polynomials. We consider the restriction of $D$ to $B^{H}$. Note that $\operatorname{Irr}(H ; F)$ consists of two elements; one is the trivial $H$-module, denoted $\epsilon$, and the other is the one dimensional non-trivial $H$-module, denoted $\delta$. Let $i: B^{H} \rightarrow B$ be the inclusion as before. Then we have

$$
D_{\epsilon}=i^{*}\left(p_{1}+p_{2}\right), \quad D_{\delta}=i^{*}\left(p_{1}-p_{2}\right) .
$$

Since $p_{2} \in \operatorname{Mor}\left(V_{l}, \operatorname{Hom}\left(U_{-m}, U_{m}\right)\right)^{K}$ and $\operatorname{Hom}\left(U_{-m}, U_{m}\right)=U_{2 m}$, the degree of (any homogeneous term in) $p_{2}$ is at least $[l, 2 \mathrm{~m} ; G]$ by 1.1 . This means that

$$
D_{\epsilon} \equiv D_{\delta} \bmod \mathscr{O}\left(B^{H}\right)_{[l, 2 m ; G]} .
$$


Since $\operatorname{det} D_{\chi}=D_{\chi}$ for $\chi=\epsilon, \delta$, we have

$$
D_{\epsilon, \delta}=\left(\operatorname{det} D_{\delta}(0)\right) \operatorname{det} D_{\epsilon}-\left(\operatorname{det} D_{\epsilon}(0)\right) \operatorname{det} D_{\delta} \in \mathscr{O}\left(B^{H}\right)_{[l, 2 m ; G]}
$$

(see I.4.0 (2)). This proves (2).

Since the computation of $[l, r ; G]$ is not difficult, the above 1.2 gives a practical estimate of the ideal $\mathscr{K}$. To apply I.4.4 it remains to choose $\Phi \in$ $\operatorname{mor}(\mathbf{F}, \mathbf{S})$ with $\Phi(0)=0$. We consider two cases.

Case 1. $B=V_{1}, F=V_{m}(0 \leq 2 m \leq n), S=\mathbb{C}$. (We may assume $0 \leq 2 m \leq$ $n$ without loss of generality because $V_{m}$ is isomorphic to $V_{-m}$ and $V_{m-n}$.) Define $\Phi \in \operatorname{mor}(\mathbf{F}, \mathbf{S})$ by

$$
\Phi(a, b)=\left(b^{m}, a^{m}\right) .
$$

It is easy to see that $E_{\Phi}(T)$ is trivial for any $T \in R(\Phi)$ when $m=0$, so we assume $m \geq 1$ (hence $\Phi(0)=0$ ) in the following. Since $S=\mathbb{C}$, we identify end $(\mathbf{S})$ with $\mathscr{O}(B)^{G}$. One can see that

$$
\begin{aligned}
& R(\Phi)_{*}=\left\{T \in \mathscr{O}(B)^{G} \mid T(0)=1\right\}, \\
& \mathscr{O}(B)^{G}=\mathbb{C}[\Delta, \Omega],
\end{aligned}
$$

where $\Delta=a b$ and $\Omega=a^{n}+b^{n}$. Set $\alpha=\min \{m,[(n+1) / 2]-m\}$.

Theorem 1.3. Let $T, T^{\prime} \in R(\Phi)_{*}$. Then

(1) $E_{\Phi}(T) \cong E_{\Phi}\left(T^{\prime}\right)$ as $G$-vector bundles only if $T \equiv T^{\prime} \bmod \left(\Delta^{\alpha}, \Omega\right)$.

(2) $E_{\Phi}(T) \cong E_{\Phi}\left(T^{\prime}\right)$ as $G_{k}$-varieties $(k \geq 2)$ only if there is a non-zero constant $c \in \mathbb{C}^{*}$ such that $T \equiv c^{*} T^{\prime} \bmod \left(\Delta^{\alpha}, \Omega\right)$.

(3) When $\alpha=m$ (i.e., $n \geq 4 m-1$ ), (1) and (2) are necessary and sufficient.

Proof. (1) An elementary computation shows that

$$
[1, r ; G]= \begin{cases}n-|r| & \text { if }|r| \leq n \leq 2|r| \\ |r| & \text { if } 2|r| \leq n\end{cases}
$$

Since $0<2 m \leq n, \mathscr{K} \subset \mathscr{O}\left(B^{H}\right)_{[1,2 m ; G]}$ by 1.2. It follows from I.4.4 that $T \equiv$ $T^{\prime} \bmod \mathscr{I}$, where $\mathscr{I}$ is the ideal in $\mathscr{O}(B)^{G}$ which maps to $\mathscr{O}\left(B^{H}\right)_{[1,2 m ; G]}$ via $i^{*}$. Since $\operatorname{deg} \Omega=n \geq[1,2 m ; G]$ and $\operatorname{deg} \Delta=2$, one sees that $\mathscr{I}=\left(\Delta^{\alpha}, \Omega\right)$ by noting $\alpha=[([1,2 m ; G]+1) / 2]$.

(2) This follows from (1) and I.5.5.

(3) We have to prove the "if" part.

(i) If $T \equiv T^{\prime} \bmod \left(\Delta^{m}, \Omega\right)$, then there are $p, q \in \mathscr{O}(B)^{G}$ such that $T^{\prime}-T=p \Delta^{m}+q \Omega$. Define $A \in \operatorname{aut}(\mathbf{F} \oplus \mathbf{S})$ by

$$
A(a, b)=\left(\begin{array}{ccc}
1 & 0 & \left(p a^{m}+q b^{n-m}\right) / 2 \\
0 & 1 & \left(p b^{m}+q a^{n-m}\right) / 2 \\
0 & 0 & 1
\end{array}\right) .
$$


One can easily check that $\left(\Phi, T^{\prime}\right)=(\Phi, T) A$, which means $E_{\Phi}(T) \cong E_{\Phi}\left(T^{\prime}\right)$ as $G$-vector bundles.

(ii) If $T \equiv c^{*} T^{\prime} \bmod \left(\Delta^{m}, \Omega\right)$, then $E_{\Phi}(T) \cong E_{\Phi}\left(c^{*} T^{\prime}\right)$ as $G$-vector bundles by (i) just above, hence, as $G_{k}$-varieties. On the other hand, $E_{\Phi}\left(c^{*} T^{\prime}\right)$ $\cong E_{\Phi}\left(T^{\prime}\right)$ as $G_{k}$-varieties by I.5.6.

Case 2. $B=V_{2}, F=V_{m}\left(0 \leq 2 m \leq n\right.$ and $m$ is odd), $S=V_{1}$. (The assumption on $m$ being odd means that the actions on elements in $\operatorname{Vec}(B, F ; S)$ are effective.) Define $\Phi \in \operatorname{mor}(\mathbf{F}, \mathbf{S})$ by

$$
\Phi(a, b)=\left(\begin{array}{cc}
b^{k} & 0 \\
0 & a^{k}
\end{array}\right)
$$

where $k=(m-1) / 2$. It is easy to see that $E_{\Phi}(T)$ is trivial for any $T \in R(\Phi)$ when $k=0$; so we assume $k \geq 1$ (hence $\Phi(0)=0$ ) in the following. Set

One can see that

$$
N= \begin{cases}n & \text { if } n \text { is odd } \\ n / 2 & \text { if } n \text { is even. }\end{cases}
$$

$$
\mathscr{O}(B)^{G}=\mathbb{C}[\Delta, \Omega]
$$

where $\Delta=a b$ as before and $\Omega=a^{N}+b^{N}$. Define $\xi_{i} \in \operatorname{end}(\mathbf{S})$ by $\xi_{0}=1$ and

$$
\xi_{1}(a, b)=\left(\begin{array}{ll}
0 & a \\
b & 0
\end{array}\right), \xi_{2}(a, b)=\left(\begin{array}{cc}
0 & b^{N-1} \\
a^{N-1} & 0
\end{array}\right), \xi_{3}(a, b)=\left(\begin{array}{cc}
b^{N} & 0 \\
0 & a^{N}
\end{array}\right) \text {. }
$$

As can easily be checked (cf. the proof of 1.1 and 1.2), any element $T$ in end $(\mathbf{S})$ is uniquely expressed as $T=\sum_{i=0}^{3} T_{(i)} \xi_{i}$ with $T_{(i)} \in \mathscr{O}(B)^{G}$. Set

$$
\beta= \begin{cases}{[(n-2 m) / 4]} & \text { if } 2 m \leq n \leq 4 m \text { and } n \text { is even, } \\ (m-1) / 2 & \text { if } 2 m \leq n \leq 4 m \text { and } n \text { is odd, or } 4 m \leq n .\end{cases}
$$

Theorem 1.4. Let $T, T^{\prime} \in R(\Phi)_{*}$. Then

(1) $E_{\Phi}(T) \cong E_{\Phi}\left(T^{\prime}\right)$ as $G$-vector bundles only if

$$
T_{(0)}^{\prime} T_{(1)} \equiv T_{(0)} T_{(1)}^{\prime} \bmod \left(\Delta^{\beta}, \Omega\right) .
$$

(2) Suppose $n$ is even. Then $E_{\Phi}(T) \cong E_{\Phi}\left(T^{\prime}\right)$ as $G$-varieties only if there is a non-zero constant $c \in \mathbb{C}^{*}$ such that

$$
\left(c^{*} T_{(0)}^{\prime}\right) T_{(1)} \equiv c\left(c^{*} T_{(1)}^{\prime}\right) T_{(0)} \quad \bmod \left(\Delta^{\beta}, \Omega\right) .
$$

Let $V \subset \mathscr{O}(B)^{G}$ be the vector space spanned by $\Delta^{i}$ for $i<\beta$. It follows from 1.4 that this vector space is mapped injectively into

$$
U=\left\{E_{\Phi}(T) \in \operatorname{VEC}(B, F ; S) \mid T \in R(\Phi)_{*}, \quad T_{(0)}=1\right\}
$$

by sending $f \in V$ to $E_{\Phi}\left(1+f \xi_{1}\right)$. Thus $1.4(1)$ implies that $U$ contains a vector space of dimension $\beta$. In addition, if $n$ is even, 1.4(2) implies that when $\beta \geq 1$, the actions defined by $E_{\Phi}\left(1+T_{(1)} \xi_{1}\right)$ and $E_{\Phi}\left(1+T_{(1)}^{\prime} \xi_{1}\right)$ are equivalent only if $T_{(1)}$ is in the orbit of $T_{(1)}^{\prime}$ under the $\mathbb{C}^{*}$-action on $V$ defined by sending $f$ to $c\left(c^{*} f\right)$ for $f \in V$ and $c \in \mathbb{C}^{*}$; so the isomorphism classes of these actions are parametrized by the orbit space $V / \mathbb{C}^{*}$ of dimension $\beta-1$. Hence 
Corollary 1.5. Let $n$ be even. If $n \geq 10$, then there is a non-linearizable action of $D_{n}$ on $\mathbb{C}^{4}$. If $n \geq 18$, then there is a family of non-linearizable actions of $D_{n}$ on $\mathbb{C}^{4}$.

Proof of Theorem 1.4. (1) An elementary computation shows that if $r$ is even, then

$$
[2, r ; G]= \begin{cases}(n-|r|) / 2 & \text { if }|r| \leq n \leq 2|r| \text { and } n \text { is even, } \\ |r| / 2 & \text { if }|r| \leq n \leq 2|r| \text { and } n \text { is odd, or } 2|r| \leq n\end{cases}
$$

Hence the integer $e$ in 1.2 is equal to $\min \{m-1,[2,2 m ; G]\}$.

Let $\epsilon$ (resp. $\delta$ ) denote the one dimensional trivial (resp. non-trivial) $H$ module as before. Let $w$ denote the coordinate of $B^{H}$ such that $w=i^{*} a=$ $i^{*} b$. Then we have

$$
\begin{aligned}
& T_{\epsilon}=i^{*} T_{(0)}+i^{*} T_{(1)} w+i^{*} T_{(2)} w^{N-1}+i^{*} T_{(3)} w^{N}, \\
& T_{\delta}=i^{*} T_{(0)}-i^{*} T_{(1)} w-i^{*} T_{(2)} w^{N-1}+i^{*} T_{(3)} w^{N},
\end{aligned}
$$

and similarly for $T^{\prime}$. Since $T_{\epsilon}^{\prime} T_{\delta} \equiv T_{\epsilon} T_{\delta}^{\prime} \bmod \mathscr{O}\left(B^{H}\right)_{e}$ by I.4.4 and 1.2 , the identities above show that

$$
i^{*}\left(T_{(0)}^{\prime} T_{(1)}\right) \equiv i^{*}\left(T_{(1)}^{\prime} T_{(0)}\right) \bmod \mathscr{O}\left(B^{H}\right)_{e-1}
$$

because $e=\min \{m-1,[2,2 m ; G]\} \leq N-1$. This means that $T_{(0)}^{\prime} T_{(1)} \equiv$ $T_{(1)}^{\prime} T_{(0)} \bmod \mathscr{I}$, where now $\mathscr{I}$ is the ideal in $\mathscr{O}(B)^{G}$ which maps to $\mathscr{O}\left(B^{H}\right)_{e-1}$ via $i^{*}$. One sees that $\mathscr{I}=\left(\Delta^{\beta}, \Omega\right)$ by noting that $\beta=[e / 2]$.

(2) The order two subgroup in $K$ satisfies the assumption in I.5.2. Hence it follows from I.5.5 together with the above argument that

$$
i^{*}\left(\left(c^{*} T_{(0)}^{\prime}\right) T_{(1)}\right) \equiv i^{*}\left(c\left(c^{*} T_{(1)}^{\prime}\right) T_{(0)}\right) \bmod \mathscr{O}\left(B^{H}\right)_{e-1}
$$

for some non-zero constant $c$. (Note that since $\operatorname{deg} \xi_{1}=1$, the coefficient of $\xi_{1}$ in $c^{*} T^{\prime}$ is $c\left(c^{*} T_{(1)}^{\prime}\right)$, i.e., $\left(c^{*} T^{\prime}\right)_{(1)}=c\left(c^{*} T_{(1)}^{\prime}\right)$.) This implies (2).

\section{2. $O(2)$}

Let $G=\mathbb{C}^{*} \rtimes \mathbb{Z} / 2 \mathbb{Z}$, which is isomorphic to $O(2)$. Let $V_{m}$ denote the two dimensional $G$-module with weights $m$ and $-m$ relative to the action of $\mathbb{C}^{*}$. The generator of $\mathbb{Z} / 2 \mathbb{Z}=H$ interchanges the two weight spaces. Then it turns out that the argument developed in the previous section works for $G=$ $\mathbb{C}^{*} \rtimes \mathbb{Z} / 2 \mathbb{Z}$ with little modification; in fact, the argument becomes simpler. The main difference is that $\mathscr{O}(B)^{G}=\mathbb{C}[\Delta]$ (i.e., $\Omega$ does not appear) and the elements $\xi_{2}, \xi_{3}$ of end(S) in Case 2 do not appear. In this section we give a complete ( $G$-variety) classification of elements $E_{\Phi}(T)$. We consider two cases following the previous section.

Case 1. $B=V_{1}, F=V_{m}(m \geq 0), S=\mathbb{C}$. Let $\Phi$ be the same as in Case 1 of $\S 1$. 
Theorem 2.1 ([MP2]). Let $T, T^{\prime} \in R(\Phi)_{*}$. Then

(1) $E_{\boldsymbol{\Phi}}(T) \cong E_{\boldsymbol{\Phi}}\left(T^{\prime}\right)$ as $G$-vector bundles if and only if $T \equiv T^{\prime} \bmod \left(\Delta^{m}\right)$.

(2) $E_{\Phi}(T) \cong E_{\Phi}\left(T^{\prime}\right)$ as $G$-varieties if and only if $T \equiv c^{*} T^{\prime} \bmod \left(\Delta^{m}\right)$ for some non-zero constant $c \in \mathbb{C}^{*}$.

Proof. The statement (1) is essentially proved in 1.3 (3), so we prove (2). Suppose $T \equiv c^{*} T^{\prime} \bmod \left(\Delta^{m}\right)$. Then $E_{\Phi}(T) \cong E_{\Phi}\left(c^{*} T^{\prime}\right)$ as $G$-vector bundles by (1), in particular, as $G$-varieties. Since $E_{\Phi}\left(c^{*} T^{\prime}\right) \cong E_{\Phi}\left(T^{\prime}\right)$ as $G$-varieties by I.5.6, the "if" part follows. We shail apply I.5.4 to prove the "only if" part. An elementary computation shows that $\operatorname{Mor}\left(V_{1}, V_{r}\right)^{G}$ is free and of rank one as a $\mathbb{C}[\Delta]$-module, where the generator is given by the map $(a, b) \rightarrow\left(a^{r}, b^{r}\right)$ if $r>0$. Thus it suffices to show the existence of the map $\omega$ in I.5.4. Take a non-trivial $m$ th root $\lambda$ of unity and define $\omega$ by $\omega(a, b)=\left(\lambda a, \lambda^{-1} b\right)$. This satisfies (1) and (2) in I.5.4. Now suppose that $E_{\Phi}(T) \cong E_{\Phi}\left(T^{\prime}\right)$ as $G$ varieties. Then it follows from I.5.4 and I.5.1 that there is a $c \in \operatorname{Aut}(B)^{G}$ such that $E_{\Phi}(T) \cong c^{*} E_{\Phi}\left(T^{\prime}\right)$ as $G$-vector bundles. Here $\operatorname{Aut}(B)^{G}=\mathbb{C}^{*}$ because $B=V_{1}$ and $\operatorname{Mor}\left(V_{1}, V_{1}\right)^{G}$ is a free $\mathbb{C}[\Delta]$-module generated by the identity map as noted above. Therefore $c^{*} E_{\Phi}\left(T^{\prime}\right) \cong E_{\Phi}\left(c^{*} T^{\prime}\right)$ as $G$-vector bundles by I.5.6, and hence $E_{\Phi}(T) \cong E_{\Phi}\left(c^{*} T^{\prime}\right)$. The desired congruence then follows from (1).

Case 2. $B=V_{2}, F=V_{m}$ ( $m>0$ and odd $), S=V_{1}$. Let $\Phi$ be the same as in Case 2 of $\S 1$. The proof of 2.2 is similar to that of 2.1 and is left to the reader.

Theorem 2.2. Let $T, T^{\prime} \in R(\Phi)_{*}$ and $k=(m-1) / 2$.

(1) $E_{\Phi}(T) \cong E_{\Phi}\left(T^{\prime}\right)$ as $G$-vector bundles if and only if $T_{(0)}^{\prime} T_{(1)} \equiv T_{(1)}^{\prime} T_{(0)}$ $\bmod \left(\Delta^{k}\right)$.

(2) $E_{\Phi}(T) \cong E_{\Phi}\left(T^{\prime}\right)$ as $G$-varieties if and only if $\left(c^{*} T_{(0)}^{\prime}\right) T_{(1)} \equiv c\left(c^{*} T_{(1)}^{\prime}\right) T_{(0)}$ $\bmod \left(\Delta^{k}\right)$ for some non-zero constant $c \in \mathbb{C}^{*}$.

Remark. This was announced in [MP1, 7.0, 7.1]. Previously Schwarz [S] had announced the classification of $\operatorname{VEC}(B, F ; S)$ in the two cases giving results related to 2.1(1) and 2.2(1). Subsequent to our announcement of 2.2(2), Kraft and Schwarz [KS, VII 5.4 (2)] used [MP1, 4.2] to prove the same result.

\section{MetacyClic GROUPS}

Let $n, q$ be positive integers and $\sigma$ an integer such that $\sigma^{q} \equiv 1 \bmod n$. In this section $G$ denotes the metacyclic group $M(n, q ; \sigma)$ generated by

$$
\left\{g, h \mid g^{n}=h^{q}=1, h g h^{-1}=g^{\sigma}\right\},
$$

which is a semidirect product of cyclic subgroups $K=\left\{g^{i} \mid 1 \leq i \leq n\right\}$ and $H=\left\{h^{j} \mid 1 \leq j \leq q\right\}$. Note that if $q=2$ and $\sigma=-1$, then $G$ is the dihedral group of order $2 n$. It turns out that the argument developed in $\S 1$ works for metacyclic groups to some extent. 
Let $V_{m}$ be the $q$-dimensional $G$-module defined by

$$
\begin{aligned}
& g v=\left(\zeta^{m} v_{0}, \zeta^{m \sigma} v_{1}, \ldots, \zeta^{m \sigma^{q-1}} v_{q-1}\right), \\
& h v=\left(v_{q-1}, v_{0}, v_{1}, \ldots, v_{q-2}\right),
\end{aligned}
$$

where $v=\left(v_{0}, \ldots, v_{q-1}\right) \in V_{m}=\mathbb{C}^{q}$ and $\zeta=\exp 2 \pi \sqrt{-1} / n$. In what follows we assume $q \geq 2$ and take $B=V_{l}, F=V_{m}$ and $S=\mathbb{C}$. Note that $B^{H}$ is the diagonal subspace of $B$, which is one dimensional. As in the dihedral case we introduce some notation to estimate the ideal $\mathscr{K}$.

Definition. With integers $l, r$ we associate non-negative integers

$$
\begin{aligned}
& {[l, r ; G]=\min \left\{\sum_{i=0}^{q-1} k_{i} \mid l \sum_{i=0}^{q-1} k_{i} \sigma^{i} \equiv r \bmod n, k_{i} \geq 0\right\},} \\
& (l, r ; G)=\min _{1 \leq j \leq q-1}\left\{\sum_{i=0}^{q-1} k_{i} \mid l \sum_{i=0}^{q-1} k_{i} \sigma^{i} \equiv r\left(1-\sigma^{j}\right) \bmod n, k_{i} \geq 0\right\} .
\end{aligned}
$$

If $G$ is a dihedral group, i.e., $q=2$ and $\sigma=-1$, then the above notation $[l, r ; G]$ agrees with that introduced in $\S 1$ and $(l, r ; G)=[l, 2 r ; G]$, so the following lemma is an extension of 1.2. In fact, the proof is almost same as that of 1.2 , so we leave it to the reader.

Lemma 3.1. (1) $J(S, F) \subset \mathscr{O}\left(B^{H}\right)_{[l, m ; G]}, J(F, S) \subset \mathscr{O}\left(B^{H}\right)_{[l,-m ; G]}$.

(2) $D(F ; S) \subset \mathscr{O}\left(B^{H}\right)_{(l, m ; G)}$.

Hence $\mathscr{K} \subset \mathscr{O}\left(B^{H}\right)_{e}$ where $e=\min \{[l, m ; G]+[l,-m ; G],(i, m ; G)\}$.

In order to make a qualitative statement it is necessary to compute the integer $e$ above. We do it later in one case. Before that we make a general statement.

Theorem 3.2. Let $B=V_{l}, F=V_{m}, S=\mathbb{C}$ as above. If

(1) $r l+m \equiv 0 \bmod n$ for some positive integer $r$,

(2) $\sum_{i=0}^{q-1} \sigma^{i} \equiv 0 \bmod n$,

then $\operatorname{VEC}(B, F ; S)$ contains a vector space of dimension $[(e-1) / q]$, where $e$ is the integer defined in 3.1 .

Proof. We denote the coordinate of $B$ by $x=\left(x_{0}, \ldots, x_{q-1}\right)$ and define $\Phi \in \operatorname{mor}(\mathbf{F}, \mathbf{S})$ by $\Phi(x)=\left(x_{0}^{r}, \ldots, x_{q-1}^{r}\right)$, where $r$ is the positive integer satisfying (1). This map is surjective on fibers over $x \neq 0$. Hence

$$
R(\Phi)_{*}=\left\{T \in \mathscr{O}(B)^{G} \mid T(0)=1\right\}
$$

We note that $\Delta=\prod_{i=0}^{q-1} x_{i}$ is in $\mathscr{O}(B)^{G}$ by (2). Considering elements $E_{\Phi}(T)$ for $T \in \mathbb{C}[\Delta] \subset \mathscr{O}(B)^{G}$ with $T(0)=1$, one sees that the statement follows from 3.1 and I.4.4.

Remark. Since $\operatorname{dim} B^{H}=1$, it follows from I.5.5 and the subsequent remark tht the set of inequivalent actions of $G_{k}(k \geq 2)$ obtained from elements in 
$\operatorname{VEC}(B, F ; S)$ is non-trivial if $e>q$ and uncountable if $e>2 q$. Here note that $G_{2}=M(n, q ; \sigma) \times \mathbb{Z} / 2 \mathbb{Z}$ is isomorphic to $M(2 n, q ; \sigma)$ if both $n$ and $\sigma$ are odd.

Here is an example of an explicit computation of the integer $e$ defined in 3.1.

Lemma 3.3. Suppose that $\sigma>0, n=\sum_{i=0}^{q-1} \sigma^{i}$ and $(q, \sigma-1)=1$. Then $e=m$ if $l \equiv 1-\sigma \bmod n$ and $0 \leq m \leq[\sigma / 2]$.

Proof. It suffices to prove that $[l, \pm m ; G] \geq m / 2$ and $(l, m ; G)=m$. First we prove $[l, m ; G] \geq m / 2$. Since $l \equiv 1-\sigma \bmod n$ and $\sum_{i=0}^{q-1} \sigma^{i} \equiv 0 \bmod n$, the congruence in the definition of $[l, m ; G]$ turns into

$$
k_{0}+\sum_{i=1}^{q-1}\left(k_{i}-k_{i-1}+m+k_{q-1}\right) \sigma^{i} \equiv 0 \bmod n .
$$

Suppose $\sum_{i=0}^{q-1} k_{i}<m / 2$. Then the coefficients of $\sigma^{i}(0 \leq i \leq q-1)$ are non-negative and strictly less than $\sigma$ as $0 \leq m \leq[\sigma / 2]$, so they must be equal because $n=\sum_{i=0}^{q-1} \sigma^{i}$. In particular, $k_{0}=k_{1}-k_{0}+m+k_{q-1}$. Hence $k_{0} \geq m / 2$, which contradicts the assumption $\sum_{i=0}^{q-1} k_{i}<m / 2$. Thus $[l, m ; G] \geq m / 2$. A similar argument works for $[l,-m ; G]$.

Next we prove $(l, m ; G)=m$. Take $j=1$ in the definition of $(l, m ; G)$. Then $k_{0}=m, k_{i}=0$ for $i \geq 1$ satisfies the congruence in the definition of $(l, m ; G)$, so $(l, m ; G) \leq m$. We shall prove the opposite inequality. Note that $(n, \sigma-1)=1$, because $n \equiv q \bmod \sigma-1$ and $(q, \sigma-1)=1$ by assumption. Since $l \equiv 1-\sigma \bmod n$ and $(n, \sigma-1)=1$, we divide the congruence in the definition of $[l, m ; G]$ by $1-\sigma$ to get

$$
\sum_{i=0}^{j-1} k_{i} \sigma^{i}+\sum_{i=j}^{q-1}\left(k_{i}+m\right) \sigma^{i} \equiv 0 \bmod n .
$$

Suppose $\sum_{i=0}^{q-1} k_{i}<m$. Then all the coefficients of $\sigma^{i}$ above are non-negative and strictly less than $\sigma$ since $0 \leq m \leq[\sigma / 2]$. Therefore they are equal; in particular, $k_{0}=k_{q-1}+m$. This contradicts the assumption $\sum_{i=0}^{q-1} k_{i}<m$; hence $(l, m ; G) \geq m$.

Combining 3.2 with 3.3 , we obtain

Theorem 3.4. Suppose that $\sigma>0, n=\sum_{i=0}^{q-1} \sigma^{i}$ and $(q, \sigma-1)=1$, where $q \geq$ 2. Then $\operatorname{VEC}\left(V_{1-\sigma}, V_{m} ; \mathbb{C}\right)$ contains a vector space of dimension $[(m-1) / q]$ if $m \leq[\sigma / 2]$.

Take $m=[\sigma / 2]$ in 3.4 and use the remark after 3.2. Then

Corollary 3.5. Suppose that $q(>1)$ and $\sigma(>0)$ are odd integers such that $(q, \sigma-1)=1$, and $n=\sum_{i=0}^{q-1} \sigma^{i}$. Then there is a non-linearizable action of $M(2 n, q ; \sigma)$ on $\mathbb{C}^{2 q}$ if $\sigma \geq 2 q+3$. In fact, there is a family of non-linearizable actions of $M(2 n, q ; \sigma)$ on $\mathbb{C}^{2 q}$ if $\sigma \geq 4 q+3$. 


\section{Subgroups of $\left(\mathbb{C}^{*}\right)^{q} \rtimes S_{q}$}

Let $H$ be a subgroup of the symmetric group $S_{q}$ on $q$ letters which acts on $\left(\mathbb{C}^{*}\right)^{q}$ by permutation of factors. Let $K$ be a subgroup of $\left(\mathbb{C}^{*}\right)^{q}$ which is invariant under the action of $H$. We take $G=K \rtimes H$. Let $V_{r}$ denote the $q$-dimensional $G$-module with coordinates $v=\left(v_{1}, \ldots, v_{q}\right)$ on which $g=$ $\left(g_{1}, \ldots, g_{q}\right) \in K$ acts by $g v=\left(g_{1}^{r} v_{1}, \ldots, g_{q}^{r} v_{q}\right)$ and $H$ acts by permutation of coordinates.

In what follows $B=V_{1} \oplus V_{-1}, F=V_{m}(m>0)$ and $S=\mathbb{C}$. We denote the coordinate of $B$ by $(a, b)$, where $a=\left(a_{1}, \ldots, a_{q}\right)$ and $b=\left(b_{1}, \ldots, b_{q}\right)$.

Lemma 4.1. Suppose that $q \geq 2, K$ contains the $q$-fold product of the cyclic group of order $\geq 2 m$, and $H$ acts transitively on $q$ letters. Then

(1) $J(S, F) \subset \mathscr{O}\left(B^{H}\right)_{m}, J(F, S) \subset \mathscr{O}\left(B^{H}\right)_{m}$,

(2) $D(F ; S) \subset \mathscr{O}\left(B^{H}\right)_{2 m}$.

Hence $\mathscr{K} \subset \mathscr{O}\left(B^{H}\right)_{2 m}$.

Proof. (1) Let $\left(f_{1}, \ldots, f_{q}\right) \in \operatorname{Mor}(B, F)^{K}$ and $g=\left(1, \ldots, 1, g_{i}, 1, \ldots, 1\right)$ $\in K$, where $g_{i}$ is in the $i$ th coordinate. It follows from equivariance that $f_{i}\left(g a, g^{-1} b\right)=g_{i}^{m} f_{i}(a, b)$. By assumption there is an element $g_{i}$ of order $\geq 2 m$, so the degree of (any homogeneous term in) $f_{i}$ with respect to $a_{i}$ and $b_{i}$ must be at least $m$ (cf. 1.1). This implies that $J(S, F) \subset \mathscr{O}\left(B^{H}\right)_{m}$. The same argument works for $J(F, S)$.

(2) Let $D=\left(p_{i j}\right) \in \operatorname{end}(\mathbf{F} ; \mathbf{S})$, where the $p_{i j}$ 's are polynomials of $a$ and $b$. The same argument as in (1) shows that the degree of (any homogeneous term in) $p_{i j}$ is $\geq 2 m$ if $i \neq j$. Since $H$ acts transitively on $q$ letters, the restrictions of $p_{i i}$ to $B^{H}$ are independent of $i$, which we denote by $p$. Let $\chi \in \operatorname{Irr}(H ; F)$ and let $n_{\chi}$ be the multiplicity of $\chi$ in $F$ viewed as an $H$ module. Then $\operatorname{det} D_{\chi} \equiv p^{n_{\chi} \operatorname{dim} \chi} \bmod \mathscr{O}\left(B^{H}\right)_{2 m}$ for any $\chi \in \operatorname{Irr}(H ; F)$. By the definition of end(F; $\mathbf{S})\left(\right.$ I.4.0(1)), and the fact that $S=\mathbb{C}$, $\operatorname{det} D_{\chi}$ is a non-zero constant unless $\chi$ is trivial. Here $\operatorname{Irr}(H ; F)$ contains a non-trivial $\chi$ since $q \geq 2$. It follows that $p$ is congruent to a non-zero constant modulo $\mathscr{O}\left(B^{H}\right)_{2 m}$ and hence so is $\operatorname{det} D_{\chi}$ for any $\chi \in \operatorname{Irr}(H ; F)$. This implies (2) (see I.4.0(2)).

Define $\Phi \in \operatorname{mor}(\mathbf{F}, \mathbf{S})$ by

$$
\Phi(a, b)=\left(b_{1}^{m}, \ldots, b_{q}^{m}\right) .
$$

Then

$$
R(\Phi)_{*}=\left\{T \in \mathscr{O}(B)^{G} \mid T(a, 0)=1\right\} .
$$

Remark. If $G=\left(\mathbb{C}^{*}\right)^{q} \rtimes S_{q}$, then $\mathscr{O}(B)^{G}=\mathbb{C}\left[\sigma_{1}, \ldots, \sigma_{q}\right]$, where $\sigma_{i}$ denotes the $i$ th elementary symmetric polynomial of $a_{1} b_{1}, \ldots, a_{q} b_{q}$. Hence if $G$ is a subgroup of $\left(\mathbb{C}^{*}\right)^{q} \rtimes S_{q}$, then $\mathscr{O}(B)^{G}$ contains $\mathbb{C}\left[\sigma_{1}, \ldots, \sigma_{q}\right]$. 
Let $i: B^{H} \rightarrow B$ be the inclusion as before. The following theorem follows from 4.1, I.4.4, and the remark above.

Theorem 4.2. Let $B=V_{1} \oplus V_{-1}, F=V_{m}$ and $S=\mathbb{C}$. Suppose that $q \geq 2$, $K$ contains the q-fold product of cyclic groups of order $\geq 2 m$, and $H$ acts transitively on $q$ letters. Let $T, T^{\prime} \in R(\Phi)_{*}$. Then $E_{\Phi}(T) \cong E_{\Phi}\left(T^{\prime}\right)$ as $G$ vector bundles only if $i^{*} T \equiv i^{*} T^{\prime} \bmod \mathscr{O}\left(B^{H}\right)_{2 m}$. In particular, $\operatorname{VEC}(B, F ; S)$ contains a vector space of dimension $m-1$.

Remark. We may take $F=V_{m} \oplus V_{-m}$ instead of $V_{m}$. Then $\Phi \in \operatorname{mor}(\mathbf{F}, \mathbf{S})$ is given by

$$
\Phi(a, b)=\left(b_{1}^{m}, \ldots, b_{q}^{m}, a_{1}^{m}, \ldots, a_{q}^{m}\right)
$$

and it turns out that the same statement as 4.2 holds. In fact, the proof is almost the same as that of 4.2 .

\section{5. $S L(2)$}

So far we have considered groups $G$ of the form of a semidirect product $K \rtimes H$, where $K$ is (the product of) a cyclic group or $\mathbb{C}^{*}$ and $H$ is a nontrivial subgroup such that $\operatorname{dim} B^{H}>0$. We observed that if the order of $K$ is sufficiently large, then $\operatorname{VEC}(B, F ; S)$ can be non-trivial for a suitable $\Phi$. It turns out that our point of view is equally effective for semisimple groups $G$. One finds that unipotent elements in $G$ play the role of $K$.

As a general remark, we note that I.4.4 produces a family of equivariant vector bundles over a $G$-module $B$ for a semisimple group $G$. In this section we preview the applications of results in [MMP1] and [MMP2] which distinguish these bundles and their underlying actions on affine space by treating the case $G=S L(2)$.

Let $G=S L(2)$ act on $\mathbb{C}^{2}$ with coordinates $(X, Y)$ by

$$
g(X, Y)=(\alpha X+\gamma Y, \beta X+\delta Y) \text { for } g=\left(\begin{array}{ll}
\alpha & \beta \\
\gamma & \delta
\end{array}\right) \in G .
$$

Then $\mathbb{C}[X, Y]$ inherits an action of $G$ and the subspace $R_{n}$ of homogeneous polynomials of degree $n$ is invariant. It is known that the $G$-module $R_{n}$ is irreducible and self-dual, i.e. $R_{n}^{*}=\operatorname{Hom}\left(R_{n}, \mathbb{C}\right) \cong R_{n}$.

Let $H$ be the subgroup of $G$ consisting of diagonal matrices, which is isomorphic to $\mathbb{C}^{*}$. Let $t^{i}$ denote the one dimensional $H$-module defined by

$$
h z=\alpha^{i} z \quad \text { for } h=\left(\begin{array}{cc}
\alpha & 0 \\
0 & \alpha^{-1}
\end{array}\right) \in H, \quad z \in t^{i}(=\mathbb{C}) .
$$

Note that

$$
R_{n} \cong \bigoplus_{i=0}^{n} t^{n-2 i} \text { as } H \text {-modules }
$$

and $X^{n-i} Y^{i}$ is in the irreducible factor of $R_{n}$ corresponding to $t^{n-2 i}$.

We take $B=R_{2}, F=R_{m}$ and $S=R_{s}$, where $m-s=2 k>0$. (It is not difficult to see that $\operatorname{VEC}(B, F ; S)$ is trivial when $m-s$ is odd.) Let $\Delta \in \mathscr{O}(B)$ 
be the discriminant, i.e., $\Delta\left(a X^{2}+b X Y+c Y^{2}\right)=b^{2}-4 a c$. It is well known and easy to prove that $\mathscr{O}(B)^{G}=\mathbb{C}[\Delta]$. Remember that $\operatorname{Mor}\left(B, R_{2 n}\right)^{G}$ is a module over $\mathscr{O}(B)^{G}=\mathbb{C}[\Delta]$. The following lemma is also known and easy to prove.

Lemma 5.1. $\operatorname{Mor}\left(B, R_{2 n}\right)^{G}$ is a free $\mathbb{C}[\Delta]$-module of rank one generated by the $n$th power map. Hence Aut $(B)^{G} \cong \mathbb{C}^{*}$.

Note that $B^{H}=\{b X Y \mid b \in \mathbb{C}\}$.

Lemma 5.2. (1) $J(S, F)=J(F, S) \subset \mathscr{O}\left(B^{H}\right)_{k}$.

(2) $D(F ; S) \subset \mathscr{O}\left(B^{H}\right)_{2 k}$.

Hence $\mathscr{K} \subset \mathscr{O}\left(B^{H}\right)_{2 k}$.

Proof. (1) Since $F$ and $S$ are self-dual, $J(S, F)=J(F, S)$. It follows from the self-duality of $F$ and the Clebsch-Gordan formula that

$$
\operatorname{Hom}(F, S) \cong F^{*} \otimes S \cong R_{m} \otimes R_{s} \cong \sum_{i=0}^{s} R_{m-s+2 i} \text { as } G \text {-modules. }
$$

Since $m-s=2 k$, any (homogeneous) element in $\operatorname{Mor}(B, \operatorname{Hom}(F, S))^{G}$ has degree $\geq k$ by 5.1 , which implies (1).

(2) There are several arguments to estimate $D(F ; S)$. We shall give one. Let $D \in \operatorname{end}(\mathbf{F} ; \mathbf{S})$ and set $D^{\prime}=D-D(0)$. It follows from the $H$-equivariance that $D^{\prime}(b X Y)$ is a projection on the subspace of $F$ spanned by $X^{i} Y^{m-i}(k \leq$ $i \leq k+s)$, i.e.,

$$
D^{\prime}(b X Y)\left(X^{i} Y^{m-i}\right)= \begin{cases}P_{i}(b) X^{i} Y^{m-i} & \text { if } k \leq i \leq k+s, \\ 0 & \text { otherwise }\end{cases}
$$

with polynomials $P_{i}$.

Take a unipotent element $g=\left(\begin{array}{cc}1 & 1 / b \\ 0 & 1\end{array}\right) \in G$. Then

$$
g X=X, \quad g Y=X / b+Y .
$$

It follows from equivariance that

$$
D^{\prime}(g(b X Y))=g D^{\prime}(b X Y) g^{-1} .
$$

We look at the image of $Y^{m}$ by this map. Since $g(b X Y)=X(X+b Y)$, the left hand side of the identity above tells that the coefficients of $X^{m-j} Y^{j}$ in the image of $Y^{m}$ are polynomials of $b$. On the other hand, it follows from the right hand side of the identity above using 5.3 and 5.4 that

$$
\begin{aligned}
g D^{\prime}(b X Y) g^{-1}\left(Y^{m}\right) & =g D^{\prime}(b X Y)(-X / b+Y)^{m} \\
& =g\left(\sum_{i=k}^{k+s} P_{i}(b)\left(\begin{array}{c}
m \\
i
\end{array}\right)(-1 / b)^{i} X^{i} Y^{m-i}\right) \\
& =\sum_{i=k}^{k+s} P_{i}(b)\left(\begin{array}{c}
m \\
i
\end{array}\right)(-1 / b)^{i} X^{i}(X / b+Y)^{m-i} .
\end{aligned}
$$


Since the coefficients of $X^{m-j} Y^{j}$ must be polynomials of $b$ as remarked above, we obtain congruences

$$
\sum_{i=k}^{k+s}(-1)^{i}\left(\begin{array}{c}
m \\
i
\end{array}\right)\left(\begin{array}{c}
m-i \\
j
\end{array}\right) P_{i}(b) \equiv 0 \bmod \left(b^{m-j}\right) .
$$

Take $j$ from 0 to $s$. Then we have $s+1$ congruence equations. An elementary observation shows that the $(s+1) \times(s+1)$ matrix formed by the coefficients of $P_{i}(b)$ in the congruences above is non-singular. This implies that $P_{i}(b) \equiv 0$ $\bmod \left(b^{2 k}\right)$, hence $D(F ; S) \subset \mathscr{O}\left(B^{H}\right)_{2 k}$.

Here is a construction of $\Phi \in \operatorname{mor}(\mathbf{F}, \mathbf{S})$. Given $x \in B$, multiplication by $x^{k}$ yields an element of $\operatorname{mor}(\mathbf{S}, \mathbf{F})$, which we denote by $\Psi$, i.e., $\Psi(x)(z)=x^{k} z$ for $z \in S$. Since $F$ and $S$ are self-dual, the dual of $\Psi$ gives an element of $\operatorname{mor}(\mathbf{F}, \mathbf{S})$. This is the desired element $\Phi$. Since $\Psi(x)$ is injective when $x \neq 0, \Phi(x)$ is surjective when $x \neq 0$. Hence we have

$$
R(\Phi)_{*}=\{T \in \operatorname{end}(\mathbf{S}) \mid T(0)=1\} .
$$

Here is the data for the next theorem:

$$
\left.B=R_{2}, \quad F=R_{2 k} \quad(k>0), \quad S=\mathbb{C} \quad \text { (i.e., } s=0\right) .
$$

In this case end $(\mathbf{S})$ can be identified with $\mathscr{O}(B)^{G}=\mathbb{C}[\Delta]$ as usual, so that $R(\Phi)_{*} \subset \mathbb{C}[\Delta]$.

Theorem 5.5. Let $T, T^{\prime} \in R(\Phi)_{*}$. Then

(1) $E_{\Phi}(T) \cong E_{\Phi}\left(T^{\prime}\right)$ as $G$-vector bundles if and only if $T \equiv T^{\prime} \bmod \left(\Delta^{k}\right)$.

(2) Suppose that $k$ is even. Then $E_{\Phi}(T) \cong E_{\Phi}\left(T^{\prime}\right)$ as $G$-varieties if and only if there is a non-zero constant $c \in \mathbb{C}^{*}$ such that $T \equiv\left(c^{*} T^{\prime}\right)$ $\bmod \left(\Delta^{k}\right)$.

Proof. (1) Suppose that $E_{\Phi}(T) \cong E_{\Phi}\left(T^{\prime}\right)$ as $G$-vector bundles. Then $i^{*} T \equiv$ $i^{*} T^{\prime} \bmod \mathscr{O}\left(B^{H}\right)_{2 k}$ by 5.2 and Theorem A (or I.4.4). Since $\mathscr{O}(B)^{G}=\mathbb{C}[\Delta]$ and $i^{*}: \mathscr{O}(B)^{G} \rightarrow \mathscr{O}\left(B^{H}\right)$ is injective, it follows that $T \equiv T^{\prime} \bmod \left(\Delta^{k}\right)$, i.e., the "only if" part has been proved.

Suppose that $T \equiv T^{\prime} \bmod \left(\Delta^{k}\right)$. Then $T^{\prime}-T=P \Delta^{k}$ with some $P \in \mathbb{C}[\Delta]$. Because $\Phi \Psi \in \operatorname{end}(\mathbf{S}) \cong \mathbb{C}[\Delta]$ is non-zero homogeneous and of degree $2 k$, $\Phi \Psi=\lambda \Delta^{k}$ for some $\lambda \in \mathbb{C}^{*}$. Hence an automorphism $A$ of $\mathbf{F} \oplus \mathbf{S}$ defined by

$$
A=\left(\begin{array}{cc}
1 & \lambda^{-1} P \Psi \\
0 & 1
\end{array}\right)
$$

satisfies $\left(\Phi, T^{\prime}\right)=(\Phi, T) A$. This proves the "if" part.

(2) The "if" part is a consequence of (1) and I.5.6. We prove the "only if" part. Since $k$ is even, multiplication by -1 on $B$ satisfies the condition of the $\omega$ in I.5.4. Hence there is a $c \in \operatorname{Aut}(B)^{G}$ such that $E_{\Phi}(T) \cong c^{*} E_{\Phi}\left(T^{\prime}\right)$ as $G$-vector bundles by I.5.1. Here $\operatorname{Aut}(B)^{G}=\mathbb{C}^{*}$ by 5.1 , so $c^{*} E_{\Phi}\left(T^{\prime}\right) \cong E_{\Phi}\left(c^{*} T^{\prime}\right)$ as $G$-vector bundles by I.5.6. Thus $T \equiv c^{*} T^{\prime} \bmod \left(\Delta^{k}\right)$ by (1). 
The theorem above implies that $\operatorname{VEC}\left(R_{2}, R_{2 k} ; \mathbb{C}\right)$ contains a vector space of dimension $k-1$. Since $-1 \in G=S L(2)$ acts trivially on elements in $\operatorname{VEC}\left(R_{2}, R_{2 k} ; \mathbb{C}\right)$, the actions of $S L(2)$ on them reduce to actions of $S O(3)=$ $S L(2) / \pm 1$.

Corollary 5.6. There is a non-linearizable action of $S O(3)$ on $\mathbb{C}^{8}$. In fact, there is a family of non-linearizable actions of $S O(3)$ on $\mathbb{C}^{2 k+4}$ when $k$ is even $\geq 4$. Remark. The former statement provides a somewhat a lower dimension for a non-linearizable action of $S O(3)$ than $[\mathrm{S}, 0.11]$, and the latter is new.

Here is the data for the next theorem:

$$
\left.B=R_{2}, \quad F=R_{2 k+1} \quad(k>0), \quad S=R_{1} \quad \text { (i.e., } s=1\right) .
$$

Since $\operatorname{Hom}(S, S) \cong R_{1} \otimes R_{1} \cong R_{2} \oplus R_{0}$ as $G$-modules, it follows from 5.1 that end $(\mathbf{S})$ is free and of rank 2 as a $\mathbb{C}[\Delta]$-module with homogenous generators of degrees 0 and 1 . For $T \in R(\Phi)_{*} \subset \operatorname{end}(\mathbf{S})$ we denote by $T_{(i)}$ the coefficient of the homogeneous generator of degree $i$. The proof of 5.7 is similar to that of 5.5 and is left to the reader.

Theorem 5.7. Let $T, T^{\prime} \in R(\Phi)_{*}$. Then

(1) $E_{\Phi}(T) \cong E_{\Phi}\left(T^{\prime}\right)$ as $G$-vector bundles if and only if $T_{(i)} \equiv T_{(i)}^{\prime}$ $\bmod \left(\Delta^{k}\right)$ for $i=0,1$.

(2) $E_{\Phi}(T) \cong E_{\Phi}\left(T^{\prime}\right)$ as $G$-varieties if and only if there is a non-zero constant $c \in \mathbb{C}^{*}$ such that $T_{(i)} \equiv c^{i}\left(c^{*} T_{(i)}^{\prime}\right) \bmod \left(\Delta^{k}\right)$ for $i=0,1$.

Corollary 5.8. There is a non-linearizable action of $S L(2)$ on $\mathbb{C}^{7}$. In fact, there is a family of non-linearizable actions of $S L(2)$ on $\mathbb{C}^{2 k+5}$ for $k \geq 2$.

Remark. A version of 5.6 and 5.8 was announced in [MP1, 1.1]. In [S, 0.11] Schwarz announced the existence of a non-linearizable action of $S L(2)$ on $\mathbb{C}^{7}$. The main content of 5.8 is that there are in fact families of actions of $S L(2)$ on $\mathbb{C}^{2 k+5}$ for $k \geq 2$. Subsequent to our announcement [KS, VII (5.7)] produced a version of this.

We wish to acknowledge that discussions with Lucy Moser-Jauslin about the $S L(2)$ case have led to the application of the techniques here to (non-abelian) connected reductive groups. This work is joint with her. See [MMP1] and [MMP2].

\section{REFERENCES}

[BH1] H. Bass and W. Haboush, Linearizing certain reductive group actions, Trans. Amer. Math. Soc. 292 (1985), 463-482.

[BH2] Some equivariant K-theory of affine algebraic groups, Comm. Algebra 15 (1987), 181-217.

[H] R. Hartshorne, Algebraic geometry, Springer-Verlag, Berlin, 1977.

[Kn] F. Knop, Nichtlinearisierbare Operationen halbeinfacher Gruppen auf affinen Räumen, Invent. Math. 105 (1991), 217-220. 
[Kr] H. Kraft, G-vector bundles and the linearization problem, Group Actions and Invariant Theory (Montréal, PQ, 1988), Canad. Math. Soc. Conf. Proc., vol. 10, Amer. Math. Soc., Providence, RI, 1989, pp. 111-123.

[KS] H. Kraft and G. W. Schwarz, Reductive group actions with one-dimensional quotient, Inst. Hautes Études Sci. Publ. Math. No. 76 (1992), 1-97.

[MP1] M. Masuda and T. Petrie, Equivariant algebraic vector bundles over representations of reductive groups: Theory, Proc. Nat. Acad. Sci. U.S.A. 88 (1991), 9061-9064.

[MP2] Algebraic families of $O(2)$-actions on affine space $\mathbb{C}^{4}$, Algebraic Groups and Their Generalizations: Classical Methods, Proc. Sympos. Pure Math., vol. 56, part 1, Amer. Math. Soc., Providence, RI, 1994, pp. 347-354.

[MMP1] M. Masuda, L. Moser-Jauslin, and T. Petrie, Equivariant algebraic vector bundles over representations of reductive groups: Applications, Proc. Nat. Acad. Sci. U.S.A. 88 (1991), 9065-9066.

[MMP2] __ Equivariant algebraic vector bundles for connected reductive groups, preprint.

[MMP3] __ Equivariant algebraic vector bundles over cones with smooth one dimensional quotient, preprint.

[MMP4] _ , The equivariant Serre problem for abelian groups, preprint.

[M] H. Matsumura, Commutative algebra, 2nd ed., Benjamin-Cummings, Reading, MA, 1980.

[Q] D. Quillen, Projective modules over polynomial rings, Invent. Math. 36 (1976), 167-171.

[S] G. W. Schwarz, Exotic algebraic group actions, C. R. Acad. Sci. Paris Sér. I. Math. 309 (1989), 89-94.

Department of Mathematics, Osaka City University, Osaka, Japan

E-mail address: masuda@tansei.cc.u-tokyo.ac.jp

Department of Mathematics, Rutgers University, New Brunswick, New Jersey 08903

E-mail address: petrie@gandalf .rutgers.edu 